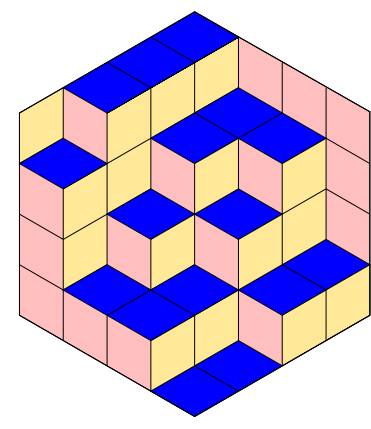

Rosa Orellana \& Mike Zabrocki

The Hopf structure of symmetric group characters as symmetric functions

Volume 4, issue 3 (2021), p. 551-574.

<http://alco.centre-mersenne.org/item/ALCO_2021__4_3_551_0>

(C) The journal and the authors, 2021.

Some rights reserved.

(c) BY This article is licensed under the

Creative Commons ATtribution 4.0 InTERnational License.

http://creativecommons.org/licenses/by/4.0/

Access to articles published by the journal Algebraic Combinatorics on the website http://alco.centre-mersenne.org/ implies agreement with the Terms of Use (http://alco.centre-mersenne.org/legal/).

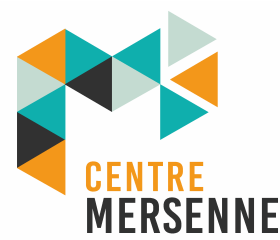

Algebraic Combinatorics is member of the Centre Mersenne for Open Scientific Publishing www.centre-mersenne.org 


\title{
The Hopf structure of symmetric group characters as symmetric functions
}

\author{
Rosa Orellana \& Mike Zabrocki
}

\begin{abstract}
In [23] the authors introduced inhomogeneous bases of the ring of symmetric functions. The elements in these bases have the property that they evaluate to characters of symmetric groups. In this article we develop further properties of these bases by proving product and coproduct formulae. In addition, we give the transition coefficients between the elementary symmetric functions and the irreducible character basis.
\end{abstract}

\section{INTRODUCTION}

In [23], the authors introduced a basis of the symmetric functions $\left\{\tilde{s}_{\lambda}\right\}$ that specialize to the characters of the irreducible modules of the symmetric group when the symmetric group $S_{n}$ is embedded in $G L_{n}$ as permutation matrices. This basis provides a new perspective on the representation theory of the symmetric group which is not well understood. In addition, several outstanding open problems in combinatorial representation theory are encoded in the linear algebra related to this basis.

One such problem is the restriction problem $[7,15,17,22,27,28]$. Since $S_{n}$ can be viewed as a subgroup of $G L_{n}$ via the embedding using permutation matrices, it follows that any irreducible polynomial module of $G L_{n}$, with character given by the Schur function $s_{\lambda}$, is a representation of $S_{n}$ via this restriction. Thus, the transition coefficients, $r_{\lambda, \mu}$, between the Schur basis $\left\{s_{\lambda}\right\}$ and the irreducible character basis $\left\{\tilde{s}_{\mu}\right\}$ encode the decomposition of a polynomial, irreducible $G L_{n}$-module into symmetric group irreducibles, i.e.

$$
s_{\lambda}=\sum_{\mu} r_{\lambda, \mu} \tilde{s}_{\mu} .
$$

In addition, we have shown in [23] that the structure coefficient of the $\left\{\tilde{s}_{\lambda}\right\}$ basis are the reduced (or stable) Kronecker coefficients, $\bar{g}_{\alpha, \beta}^{\gamma}[2,3,4,5,6,10,19,20,21]$. The history of these problems indicates that they are difficult and are unlikely to be resolved in a single step. In order to make further progress, we need to develop some of the properties of character bases so that they can be treated as familiar objects in the ring of symmetric functions.

The combinatorics related to the irreducible character basis involves multiset partitions and multiset tableaux. Developing analogues of the notion of 'lattice' that

\footnotetext{
Manuscript received 8th June 2019, revised 30th January 2021, accepted 31st January 2021.
}

KEYWORDS. symmetric functions, symmetric group characters, Hopf algebra.

ACKNOWLEDGEMENTS. Work supported by NSF grants DMS-1300512 and DMS-1700058, and by NSERC. 
exists for words and column strict tableaux would likely help find a combinatorial interpretations for

(1) the transition coefficients, $r_{\lambda, \mu}$, from the Schur function $s_{\lambda}$ to the irreducible character basis; and

(2) the structure coefficients of the irreducible character basis, $\bar{g}_{\alpha, \beta}^{\gamma}$.

The Hopf algebra structure that we develop here lays out the combinatorics and indicates that a combinatorial interpretation for the stable Kronecker and restriction problems may exist using operations on multiset tableaux.

In order to develop symmetric function expressions for irreducible characters and to develop their properties, the authors introduced in [23] an intermediate basis $\tilde{h}_{\lambda}$ which represent the characters of the trivial module in $S_{\lambda_{1}} \times S_{\lambda_{2}} \times \cdots \times S_{\lambda_{\ell(\lambda)}}$ induced to $S_{n}$. A third character basis, $\tilde{x}_{\lambda}$, was introduced in [1] and it represents the character of the module $\mathbb{S}^{\lambda} \otimes \mathbb{S}^{(n-|\lambda|)}$ of $S_{|\lambda|} \times S_{n-|\lambda|}$ induced to $S_{n}$, where $\mathbb{S}^{\lambda}$ is the irreducible module indexed by $\lambda$ and $\mathbb{S}^{(n-|\lambda|)}$ is the trivial module. Assaf and Speyer used this basis to show that the Schur expansion of the $\tilde{s}_{\lambda}$ basis is sign alternating in degree. We show that the power sum expansion of all three of the character bases is given by expanding them in terms of two analogues of the power sum basis, $\mathbf{p}_{\lambda}$ and $\overline{\mathbf{p}}_{\lambda}$ (see Equations (3)-(4)).

In [24] we gave combinatorial interpretations for some products involving the $\tilde{s}_{\lambda}$ basis. The purpose of this paper is to further develop algebraic properties of the character and related bases. The main results in this paper are product formulae for the $\tilde{h}_{\lambda}, \mathbf{p}_{\lambda}$ and $\overline{\mathbf{p}}_{\lambda}$ bases; as well as coproduct formulae for the $\tilde{h}_{\lambda}, \mathbf{p}_{\lambda}, \overline{\mathbf{p}}_{\lambda}, \tilde{x}_{\lambda}$ and $\tilde{s}_{\lambda}$ bases. The coproduct formulae correspond to restriction of characters from $S_{n}$ to $S_{r} \times S_{s}$ with $r+s=n$.

In [23] we provided a combinatorial interpretation for the transition coefficients from the complete homogeneous basis to the $\tilde{s}_{\lambda}$-basis in terms of multiset tableaux. In representation theory, this is equivalent to computing the multiplicities when we restrict the tensor product of symmetric tensors from $G L_{n}$ to $S_{n}$. In this paper, we give a combinatorial interpretation for the expansion of the elementary basis in the irreducible character basis. This corresponds to finding a combinatorial interpretation of the multiplicities of the restriction of the antisymmetric tensor products from $G L_{n}$ to $S_{n}$.

\section{Notation and Preliminaries}

The combinatorial objects that arise in our work are the classical building blocks: set, multiset, partition, set partition, multiset partition, composition, weak composition, tableau, words, etc. In this section we remind the reader about the definitions of these objects as well as establish notation and usual conventions that we will use in this paper.

A partition of a non-negative integer $n$ is a weakly decreasing sequence $\lambda=$ $\left(\lambda_{1}, \lambda_{2}, \ldots, \lambda_{\ell}\right)$ such that $|\lambda|=\lambda_{1}+\lambda_{2}+\cdots+\lambda_{\ell}=n$. We call $n=|\lambda|$ the size of $\lambda$. The $\lambda_{i}$ 's are called the parts of the partition and the number of nonzero parts is called the length and it is often denoted $\ell(\lambda)=\ell$. We use the notation $\lambda \vdash n$ to mean that $\lambda$ is a partition of $n$. We reserve $\lambda$ and $\mu$ exclusively for partitions. We denote by $m_{i}(\lambda)$ the multiplicity of $i$ in $\lambda$, that is, the number of times it occurs as a part in $\lambda$. Another useful notation for a partition is the exponential notation where $m_{i}=m_{i}(\lambda)$ and $\lambda=\left(1^{m_{1}} 2^{m_{2}} \cdots k^{m_{k}}\right)$. With this notation the number of permutations with cycle 
structure $\lambda \vdash n$ is $\frac{n !}{z_{\lambda}}$ where

$$
z_{\lambda}=\prod_{i=1}^{\lambda_{1}} m_{i}(\lambda) ! i^{m_{i}(\lambda)} .
$$

The most common operation we use is that of adding a part of size $n$ to the beginning of a partition. This is denoted $(n, \lambda)$. If $n<\lambda_{1}$, this sequence will no longer be an integer partition and we will have to interpret the object appropriately. Similarly, if $\lambda$ is non-empty, then let $\bar{\lambda}=\left(\lambda_{2}, \lambda_{3}, \ldots, \lambda_{\ell(\lambda)}\right)$.

For a partition $\lambda$ the set $\left\{(i, j): 1 \leqslant i \leqslant \lambda_{j}, 1 \leqslant j \leqslant \ell(\lambda)\right\}$ are called the cells of $\lambda$ and we represent these cells as stacks of boxes in the first quadrant with the largest part at the bottom following the 'French notation'. This graphical representation of the cells is called the Young diagram of $\lambda$. A tableau is a mapping from the set of cells to a set of labels. A tableau will be represented by filling the boxes of the diagram of a partition with the labels. In our case, we will encounter tableaux where only a subset of the cells are mapped to a label. The content of a tableau is the multiset obtained with the total number of occurrences of each number.

A multiset is a collection of elements such that repetitions are allowed, multisets are denoted by $\left\{\left\{b_{1}, b_{2}, \ldots, b_{r}\right\}\right.$. Multisets will also be represented by exponential notation so that $\left\{\left\{1^{a_{1}}, 2^{a_{2}}, \ldots, \ell^{a_{\ell}}\right\}\right\}$ represents the multiset where the value $i$ occurs $a_{i}$ times.

A set partition of a set $S$ is a set of subsets $\left\{S_{1}, S_{2}, \ldots, S_{\ell}\right\}$ with $S_{i} \subseteq S$ for $1 \leqslant i \leqslant \ell, S_{i} \cap S_{j}=\varnothing$ for $1 \leqslant i<j \leqslant \ell$ and $S_{1} \cup S_{2} \cup \cdots \cup S_{\ell}=S$. A multiset partition $\pi=\left\{\left\{S_{1}, S_{2}, \ldots, S_{\ell}\right\}\right\}$ of a multiset $S$ is a similar construction to a set partition, but now $S_{i}$ is a multiset, and it is possible that two multisets $S_{i}$ and $S_{j}$ have non-empty intersection (and may even be equal). The length of a multiset partition is the number of non-empty multisets in the partition and it is denoted by $\ell(\pi)=\ell$. We will use the notation $\pi+S$ to indicate that $\pi$ is a multiset partition of the multiset $S$.

We will use $\tilde{m}(\pi)$ to represent the partition of $\ell(\pi)$ consisting of the multiplicities of the multisets which occur in $\pi$ (e.g. $\tilde{m}(\{\{\{1,1,2\}\},\{1,1,2\},\{\{1,3\}\}\})=(2,1)$ because $\{\{1,1,2\}$ occurs 2 times and $\{1,3\}$ occurs 1 time).

For non-negative integers $n$ and $\ell$, a composition of size $n$ is an ordered sequence of positive integers $\alpha=\left(\alpha_{1}, \alpha_{2}, \ldots, \alpha_{\ell}\right)$ such that $\alpha_{1}+\alpha_{2}+\cdots+\alpha_{\ell}=n$. A weak composition is such a sequence with the condition that $\alpha_{i} \geqslant 0$ (zeros are allowed). To indicate that $\alpha$ is a composition of $n$ we will use the notation $\alpha \models n$ and to indicate that $\alpha$ is a weak composition of $n$ we will use the notation $\alpha \models_{w} n$. For both compositions and weak compositions, $\ell(\alpha):=\ell$.

2.1. The Ring of SyMmetric FUnCtions. For some modern references on this subject see for example $[16,18,25,30]$. The ring of symmetric functions will be denoted Sym $=\mathbb{Q}\left[p_{1}, p_{2}, p_{3}, \ldots\right]$. The $p_{k}$ are power sum generators and they will be thought of as functions which can be evaluated at values when appropriate by making the substitution $p_{k} \rightarrow x_{1}^{k}+x_{2}^{k}+\ldots+x_{n}^{k}$ but they will be used algebraically in this ring without reference to their variables. Thus the degree of each $p_{k}$ is $k$. It is well-known that the monomials in the power sums $p_{\lambda}:=p_{\lambda_{1}} p_{\lambda_{2}} \cdots p_{\lambda_{\ell(\lambda)}}$ where $\lambda \vdash n$ span the subspace of the symmetric functions of degree $n$.

For any symmetric function $f \in$ Sym, $f$ is a linear combination of the power sum basis $f=\sum_{\lambda} a_{\lambda} p_{\lambda}$, then for any expression $E\left(x_{1}, x_{2}, x_{3}, \ldots\right)$ in the set of variables $x_{1}, x_{2}, x_{3}, \ldots$ (and potentially $y_{1}, y_{2}, y_{3}, \ldots, z_{1}, z_{2}, z_{3}, \ldots$ ) the notation $f[E]$ will represent

$$
f\left[E\left(x_{1}, x_{2}, x_{3}, \ldots\right)\right]=\sum_{\lambda} a_{\lambda} \prod_{i=1}^{\ell(\lambda)} E\left(x_{1}^{\lambda_{i}}, x_{2}^{\lambda_{i}}, x_{3}^{\lambda_{i}}, \ldots\right)
$$


The advantage of this notation is that if $X_{n}=x_{1}+x_{2}+\cdots+x_{n}$, then $f\left[X_{n}\right]$ is a symmetric polynomial in $n$ variables since $p_{k}\left[X_{n}\right]=x_{1}^{k}+x_{2}^{k}+\cdots+x_{n}^{k}$ is the power sum symmetric polynomial. This notation is also practical because by defining $X=x_{1}+x_{2}+\cdots$, then $f[X]$ is a symmetric series in the set of variables $x_{1}, x_{2}, x_{3}, \ldots$ such that if the variables $x_{i}=0$ for $i>n$, then the resulting expression is $f\left[X_{n}\right]$. For a given alphabet $X$, set $\operatorname{Sym}_{X}$ to be the symmetric functions (or symmetric polynomials if $X$ is finite) in the variables $X$ and $\operatorname{Sym}_{X} \simeq$ Sym as a graded Hopf algebra.

A Hopf algebra [32] is a vector space with both a product and a coproduct that form a bialgebra structure along with some additional relations including the existence of the map known as an antipode. Hopf algebras arise in many areas of mathematics, but in algebraic combinatorics the symmetric functions as a graded bialgebra is one of the prototypes for what is known as a 'combinatorial Hopf algebra' [13].

The symmetric functions have a Hopf structure on it, [13, Chapter 2], and the coproduct operation can be encoded in operations of evaluating the symmetric function on sets of variables. The $p_{k}$ are primitive elements of Sym so that $\Delta\left(p_{k}\right)=$ $p_{k} \otimes 1+1 \otimes p_{k}$. Since $p_{k}[X+Y]=p_{k}[X]+p_{k}[Y]$, it follows that if we represent the coproduct of $f$ in Sweedler notation, $\Delta(f)=\sum_{i} f^{(i)} \otimes \tilde{f}^{(i)}$, then it is possible to derive,

$$
f[X+Y]=\sum_{i} f^{(i)}[X] \tilde{f}^{(i)}[Y]
$$

by linearity relations calculated on the power sum basis. We will calculate formulas for the coproduct operation on the character bases in Section 4 and this symmetric function notation will be helpful in our proofs since $\operatorname{Sym} \otimes \operatorname{Sym} \simeq \operatorname{Sym}_{X, Y}$ as a graded Hopf algebra.

The standard bases of Sym (each indexed by the set of partitions $\lambda$ ) are power sum $\left\{p_{\lambda}\right\}_{\lambda}$, homogeneous/complete $\left\{h_{\lambda}\right\}_{\lambda}$, elementary $\left\{e_{\lambda}\right\}_{\lambda}$, monomial $\left\{m_{\lambda}\right\}_{\lambda}$, and Schur $\left\{s_{\lambda}\right\}_{\lambda}$. The Hall inner product is defined by declaring that the power sum basis is orthogonal, i.e. $\left\langle\frac{p_{\lambda}}{z_{\lambda}}, p_{\mu}\right\rangle=\delta_{\lambda \mu}$, where we use the notation $\delta_{\lambda \mu}=1$ if $\lambda=\mu$ and 0 if $\lambda \neq \mu$. Under this inner product the Schur functions are orthonormal and the monomial and homogeneous functions are dual, i.e. $\left\langle s_{\lambda}, s_{\mu}\right\rangle=\left\langle h_{\lambda}, m_{\mu}\right\rangle=\delta_{\lambda \mu}$. We use this scalar product to represent values of coefficients by taking scalar products with dual bases. In particular, an identity that we will repeatedly use

$$
f=\sum_{\lambda}\left\langle f, a_{\lambda}\right\rangle b_{\lambda}
$$

for any pair of bases $\left\{a_{\lambda}\right\}_{\lambda}$ and $\left\{b_{\lambda}\right\}_{\lambda}$ such that $\left\langle a_{\lambda}, b_{\mu}\right\rangle=\delta_{\lambda \mu}$.

The scalar product on Sym naturally extends to a scalar product on $\mathrm{Sym}_{X}$, $\operatorname{Sym} \otimes \operatorname{Sym}$ and $\operatorname{Sym}_{X, Y}$. The identities that we will use to calculate coproducts on the character symmetric functions in Section 4 can be found in $[18$, Section 5 , p. 91-92].

We will also refer to the irreducible character of the symmetric group indexed by the partition $\lambda$ and evaluated at a permutation of cycle structure $\mu$ as the coefficient $\left\langle s_{\lambda}, p_{\mu}\right\rangle=\chi^{\lambda}(\mu)$. For $k>0$, define

$$
\Xi_{k}:=1, \mathrm{e}^{2 \pi \mathrm{i} / k}, \mathrm{e}^{4 \pi \mathrm{i} / k}, \ldots, \mathrm{e}^{2(k-1) \pi \mathrm{i} / k}
$$

as a symbol representing the eigenvalues of a permutation matrix of a $k$-cycle. Then for any partition $\mu$, let

$$
\Xi_{\mu}:=\Xi_{\mu_{1}}, \Xi_{\mu_{2}}, \ldots, \Xi_{\mu_{\ell(\mu)}}
$$

be the multiset of eigenvalues of a permutation matrix with cycle structure $\mu$. We will evaluate symmetric functions at these eigenvalues. The notation $f\left[\Xi_{\mu}\right]$ represents taking the element $f \in$ Sym and replacing $p_{k}$ in $f$ with $x_{1}^{k}+x_{2}^{k}+\cdots+x_{|\mu|}^{k}$ and 
then replacing the variables $x_{i}$ with the values in $\Xi_{\mu}$. In particular, for $r, k>0$, $p_{r}\left[\Xi_{k}\right]=0$ unless either $r=0$ (in which case the value is 1 ) or $k$ divides $r$ (in which case $\left.p_{r}\left[\Xi_{k}\right]=k\right)$.

From [23], a useful tool that we will repeatedly use to establish symmetric function identities is the following proposition.

Proposition 2.1 ([23, Proposition 38] and [23, Corollary 40]). Let $f, g \in$ Sym be symmetric functions of degree less than or equal to some positive integer $n$. Assume that

$$
f\left[\Xi_{\gamma}\right]=g\left[\Xi_{\gamma}\right]
$$

for all partitions $\gamma$ such that $|\gamma| \leqslant n$ (respectively, $|\gamma| \geqslant n$ ), then $f=g$ as elements of Sym.

2.2. Symmetric Group CharaCters AS SYMmetric FUnCtions. In this section, we will define five additional bases for the ring of symmetric functions and discuss their connections to the characters of the symmetric group. Four of these appeared in [23] and the fifth basis was introduced in [1].

The following expressions were developed from character polynomials and yield two analogues of the power sum basis. For each $i, r>0$, define

$$
\overline{\mathbf{p}}_{i^{r}}=i^{r}\left(\frac{1}{i} \sum_{d \mid i} \mu(i / d) p_{d}\right)_{r} \text { and } \mathbf{p}_{i^{r}}=\sum_{k=0}^{r}(-1)^{r-k}\left(\begin{array}{l}
r \\
k
\end{array}\right) \overline{\mathbf{p}}_{i^{k}}
$$

where $(x)_{k}=x(x-1) \cdots(x-k+1)$ denotes the $k$-th falling factorial. Then if a partition $\gamma$ is expressed as $\gamma=1^{m_{1}(\gamma)} 2^{m_{2}(\gamma)} \cdots \ell^{m_{\ell}(\gamma)}$, we set

$$
\overline{\mathbf{p}}_{\gamma}:=\prod_{i \geqslant 1} \overline{\mathbf{p}}_{i^{m_{i}(\gamma)}} \text { and } \mathbf{p}_{\gamma}:=\prod_{i \geqslant 1} \mathbf{p}_{i^{m_{i}(\gamma)}} .
$$

Note that by Möbius inversion and Equation (3) we also have that

$$
\overline{\mathbf{p}}_{i^{r}}=\sum_{k=0}^{r}\left(\begin{array}{l}
r \\
k
\end{array}\right) \mathbf{p}_{i^{k}}
$$

Now define three more bases,

(4) $\quad \tilde{s}_{\lambda}=\sum_{\gamma \vdash|\lambda|} \chi^{\lambda}(\gamma) \frac{\mathbf{p}_{\gamma}}{z_{\gamma}}, \quad \tilde{x}_{\lambda}=\sum_{\gamma \vdash|\lambda|} \chi^{\lambda}(\gamma) \frac{\overline{\mathbf{p}}_{\gamma}}{z_{\gamma}} \quad$ and $\quad \tilde{h}_{\lambda}=\sum_{\gamma \vdash|\lambda|}\left\langle h_{\lambda}, p_{\gamma}\right\rangle \frac{\overline{\mathbf{p}}_{\gamma}}{z_{\gamma}}$.

We refer to the set of functions $\tilde{s}_{\lambda}$ as the irreducible character basis since $\tilde{s}_{\lambda}\left[X_{n}\right]$ are symmetric polynomials which evaluate to the irreducible characters of the symmetric group in the same way that Schur functions evaluate to the irreducible characters of polynomial $G L_{n}$ modules. Speyer and Assaf [1] used the notation $s_{\lambda}^{\dagger}$ for $\tilde{s}_{\lambda}$ and called them "stable Specht polynomials." The foundations of these symmetric functions is likely found in earlier work than that of Specht [29] on the representation theory of the symmetric groups and go back to Frobenius [11] and Young [33]. We refer to the set of functions $\tilde{x}_{\lambda}$ as the induced irreducible character basis since, for $n \geqslant$ $|\lambda|+\lambda_{1}$, it encodes the character of the module $\mathbb{S}^{\lambda} \otimes \mathbb{S}^{(n-|\lambda|)} \uparrow_{S_{|\lambda|} \times S_{n-|\lambda|}}^{S_{n}}$, where $\mathbb{S}^{\lambda}$ is the irreducible module indexed by $\lambda$ and $\mathbb{S}^{(n-|\lambda|)}$ is the trivial module of $S_{n-|\lambda|}$. As a symmetric function, $\tilde{x}_{\lambda}$ is a sum of irreducible character basis elements with nonnegative coefficients. We refer to the set of functions $\tilde{h}_{\lambda}$ as the induced trivial character basis since the $\tilde{h}_{\lambda}\left[\Xi_{\mu}\right]$ are values of trivial characters induced from Young subgroups $S_{\lambda_{1}} \times \cdots \times S_{\lambda_{r}}$ to the symmetric group $S_{n}$.

Theorem 14 and Equations (34) and (35) from [23] say that if $\lambda$ is a partition and $n$ is an integer such that $n \geqslant|\lambda|+\lambda_{1}$ and $\mu$ is a partition of $n$, then $\tilde{s}_{\lambda}\left[\Xi_{\mu}\right]=$ 
$\chi^{(n-|\lambda|, \lambda)}(\mu)$. Proposition 16 and Lemma 15 of [23] says that $\tilde{h}_{\lambda}\left[\Xi_{\mu}\right]=\left\langle h_{(n-|\lambda|, \lambda)}, p_{\mu}\right\rangle$ if $n \geqslant|\lambda|$ and 0 otherwise. It is well-known that $\left\langle h_{\mu}, p_{\gamma}\right\rangle=\sum_{\lambda \vdash|\mu|} K_{\lambda \mu}\left\langle s_{\lambda}, p_{\gamma}\right\rangle$, where $K_{\lambda \mu}$ are the Kostka coefficients. Then, it follows that $\tilde{h}_{\mu}=\sum_{\lambda \vdash|\mu|} K_{\lambda \mu} \tilde{x}_{\lambda}$ and $\tilde{x}_{\lambda}\left[\Xi_{\mu}\right]=\left\langle s_{(n-|\lambda|)} s_{\lambda}, p_{\mu}\right\rangle$ if $n \geqslant|\lambda|$ and 0 otherwise. by

As Assaf and Speyer [1, Proposition 5] point out, the $\tilde{x}_{\lambda}$ and $\tilde{s}_{\lambda}$ bases are related

$$
\tilde{x}_{\lambda}=\sum_{\nu} \tilde{s}_{\nu} \quad \text { and } \quad \tilde{s}_{\lambda}=\sum_{\nu}(-1)^{|\lambda / \nu|} \tilde{x}_{\nu}
$$

where the sum on the left (resp. sum on the right) is over partitions $\nu$ contained in $\lambda$ such that $\lambda / \nu$ is a horizontal strip (resp. vertical strip). That is, if the Young diagram for $\nu$ is drawn inside the Young diagram for $\lambda$, then there is at most one cell per column (resp. row) in $\lambda$ which is not in $\nu$.

The $\tilde{s}_{\lambda}$ form a basis of the symmetric functions with the property that $\tilde{s}_{\lambda}\left[X_{n}\right]$ is a symmetric polynomial (of inhomogeneous degree) that evaluates to the characters of the irreducible symmetric group modules $\mathbb{S}^{(n-|\lambda|, \lambda)}$. Then, the character symmetric functions are a means of encoding the characters of families of symmetric group modules which are representation stable (a notion formalized in [9]). They are equivalent to finitely generated FI-modules, a category introduced by Church, Ellenberg and Farb in [8] to capture the fundamental properties of representation theory stability.

Let $F I$ be the category with objects $[n]:=\{1,2, \ldots, n\}$ and whose morphisms are injections $[n] \hookrightarrow[m]$. An FI-module is a functor $V$ from $F I$ to the category of $\mathbb{C}$-modules. By evaluation at a set $[n]$, the functor $V$ realizes a family of $S_{n}$ modules $V([n])$. The character basis is a way of encoding the character of this family as a symmetric function.

In particular, Theorem 3.3.4 of [8] shows that that if $V$ is a finitely generated FI-module, then there a polynomial $P_{V}\left(X_{1}, X_{2}, \ldots\right)$ such that the character of a permutation $\sigma$ (of a sufficiently large $n$ ) acting on the module $V([n])$ is an evaluation of $P_{V}$. This implies by Proposition 12 of [23] that there is a corresponding symmetric function $f_{V}$ such that if the permutation $\sigma$ has cycle structure $\mu$, then the character of $\sigma$ acting on $V([n])$ is equal to $f_{V}\left[\Xi_{\mu}\right]$.

Summarizing Theorem 3.3.4 appearing in [8] and how they apply to the irreducible character basis, we have the following proposition.

Proposition 2.2. For every finitely generated FI-module $V$, there exists a positive integer $M$ and coefficients $a_{\lambda}$ if and only if for all $n \geqslant M$,

$$
V([n]) \simeq \bigoplus_{\lambda}\left(\mathbb{S}^{(n-|\lambda|, \lambda)}\right)^{\oplus a_{\lambda}}
$$

and

$$
f_{V}=\sum_{\lambda} a_{\lambda} \tilde{s}_{\lambda}
$$

is a symmetric function such that $f_{V}\left[\Xi_{\mu}\right]$ is the character of a permutation $\sigma$ of cycle structure $\mu \vdash n$ acting on $V([n])$.

2.3. A scalar product on Characters and the Frobenius map. In [24] we introduced a scalar product on symmetric functions (coming from the usual scalar product on irreducible characters of the symmetric group) for which the irreducible character basis was orthogonal. This scalar product was useful in the application presented in that paper because it allowed us to calculate an individual coefficient of an irreducible character in an expression. In this section we develop some of the properties of this scalar product further. 
For all $n$ sufficiently large,

$$
\sum_{\nu \vdash n} \frac{\tilde{s}_{\lambda}\left[\Xi_{\nu}\right] \tilde{s}_{\mu}\left[\Xi_{\nu}\right]}{z_{\nu}}=\sum_{\nu \vdash n} \frac{\chi^{(n-|\lambda|, \lambda)}(\nu) \chi^{(n-|\mu|, \mu)}(\nu)}{z_{\nu}}=\delta_{\lambda \mu} .
$$

Now the right hand side of this expression is independent of $n$. Thus, for a sufficiently large $n$ and for any symmetric functions $f$ and $g$, the expression $\sum_{\nu \vdash n} \frac{f\left[\Xi_{\nu}\right] g\left[\Xi_{\nu}\right]}{z_{\nu}}$ is also independent of $n$ by linearity since $\left\{\tilde{s}_{\lambda}\right\}$ is a basis.

Therefore we may define

$$
\langle f, g\rangle_{@}=\sum_{\nu \vdash n} \frac{f\left[\Xi_{\nu}\right] g\left[\Xi_{\nu}\right]}{z_{\nu}}=\frac{1}{n !} \sum_{\sigma \in S_{n}} f\left[\Xi_{c y c(\sigma)}\right] g\left[\Xi_{c y c(\sigma)}\right] .
$$

for $n \geqslant 2 \max (\operatorname{deg}(f), \operatorname{deg}(g))$ and where $\operatorname{cyc}(\sigma)$ is a partition representing the cycle structure of $\sigma \in S_{n}$. We use the @-symbol as a subscript of the right angle bracket to differentiate this scalar product from the usual scalar product where $\left\langle s_{\lambda}, s_{\mu}\right\rangle=\delta_{\lambda \mu}$.

We can relate these scalar products by using the Frobenius map which is a linear isomorphism from the class functions of the symmetric group to the ring of symmetric functions. Since we know that characters of the symmetric group (and hence class functions) can be expressed as symmetric functions, we can define the Frobenius map or characteristic map on symmetric functions

$$
\phi_{n}(f)=\sum_{\nu \vdash n} f\left[\Xi_{\nu}\right] \frac{p_{\nu}}{z_{\nu}} .
$$

We have that $\phi_{n}$ is a map from the ring of symmetric functions to the subspace of symmetric functions of degree $n$. And $\phi_{n}$ has the property that for symmetric functions $f$ and $g$,

$$
\phi_{n}(f g)=\phi_{n}(f) * \phi_{n}(g),
$$

where $*$ denotes the Kronecker (or internal) product of symmetric functions. Since $\tilde{h}_{\lambda}\left[\Xi_{\nu}\right]=\left\langle h_{|\nu|-|\lambda|} h_{\lambda}, p_{\nu}\right\rangle, \tilde{x}_{\lambda}\left[\Xi_{\mu}\right]=\left\langle s_{(n-|\lambda|)} s_{\lambda}, p_{\mu}\right\rangle$ and $\tilde{s}_{\lambda}\left[\Xi_{\nu}\right]=\chi^{(|\nu|-|\lambda|, \lambda)}(\nu)$ if $|\nu| \geqslant|\lambda|+\lambda_{1}$, then the image of $\tilde{s}_{\lambda}, \tilde{x}_{\lambda}$ and $\tilde{h}_{\lambda}$ are

$$
\phi_{n}\left(\tilde{s}_{\lambda}\right)=s_{(n-|\lambda|, \lambda)}, \quad \phi_{n}\left(\tilde{x}_{\lambda}\right)=s_{(n-|\lambda|)} s_{\lambda} \quad \text { and } \quad \phi_{n}\left(\tilde{h}_{\lambda}\right)=h_{(n-|\lambda|, \lambda)} .
$$

This leads to the following proposition.

Proposition 2.3. If $n \geqslant 2 \max (\operatorname{deg}(f), \operatorname{deg}(g))$, then

$$
\langle f, g\rangle_{@}=\left\langle\phi_{n}(f), \phi_{n}(g)\right\rangle .
$$

Proof. For partitions $\lambda$ and $\mu$, take an $n$ which is sufficiently large (take $n \geqslant$ $2 \max (|\lambda|,|\mu|))$, then $(n-|\lambda|, \lambda)$ and $(n-|\mu|, \mu)$ are both partitions and this scalar product can easily be computed on the irreducible character basis by

$$
\left\langle\phi_{n}\left(\tilde{s}_{\lambda}\right), \phi_{n}\left(\tilde{s}_{\mu}\right)\right\rangle=\left\langle s_{(n-|\lambda|, \lambda)}, s_{(n-|\mu|, \mu)}\right\rangle=\delta_{\lambda \mu}=\left\langle\tilde{s}_{\lambda}, \tilde{s}_{\mu}\right\rangle_{@} .
$$

Since this calculation holds on a basis, Equation (9) holds for all symmetric functions $f$ and $g$ by linearity.

The $\tilde{s}_{\lambda}$ symmetric functions are the orthonormal basis with respect to the scalar product $\langle\cdot, \cdot\rangle_{@}$. This basis is triangular with respect the Schur basis $\left(\tilde{s}_{\lambda}\right.$ is equal to $s_{\lambda}$ plus terms of lower degree) and hence it may be calculated using Gram-Schmidt orthonormalization with respect to the @-scalar product.

REMARK 2.4. Using the fact that $\tilde{s}_{\lambda}$ is an orthonormal basis and $s_{\lambda}=\sum_{\gamma} r_{\lambda, \gamma} \tilde{s}_{\gamma}$ where $r_{\lambda, \gamma}$ are the multiplicities in the decomposition of a polynomial, irreducible $G L_{n}$-module into symmetric group irreducibles. We have that $\left\langle s_{\lambda}, s_{\mu}\right\rangle_{@}=\sum_{\gamma} r_{\lambda, \gamma} r_{\mu, \gamma}$ which is a positive integer. 
The analogue $\mathbf{p}_{\lambda}$ of power sums are orthogonal with respect to the @-scalar product in the same way that the power sums are orthogonal with respect to the Hall scalar product. By the definition, Equation (4),

$$
\mathbf{p}_{\lambda}=\sum_{\mu \vdash|\lambda|}\left\langle p_{\lambda}, s_{\mu}\right\rangle \tilde{s}_{\mu} .
$$

Proposition 2.5. For all partitions $\lambda$ and $\mu$,

$$
\left\langle\mathbf{p}_{\lambda}, \mathbf{p}_{\mu}\right\rangle_{@}=z_{\lambda} \delta_{\lambda \mu} \text {. }
$$

Proof. By Equation (10),

$$
\left\langle\mathbf{p}_{\lambda}, \mathbf{p}_{\mu}\right\rangle_{@}=\sum_{\gamma \vdash|\lambda|} \sum_{\nu \vdash|\mu|}\left\langle p_{\lambda}, s_{\gamma}\right\rangle\left\langle p_{\mu}, s_{\nu}\right\rangle\left\langle\tilde{s}_{\gamma}, \tilde{s}_{\nu}\right\rangle_{@}=\sum_{\nu \vdash|\mu|}\left\langle p_{\lambda}, s_{\nu}\right\rangle\left\langle p_{\mu}, s_{\nu}\right\rangle .
$$

This expression is equal to 0 if $|\lambda|$ is not equal to $|\mu|$. If they are equal, the right hand side is equal to $\left\langle p_{\lambda}, p_{\mu}\right\rangle=z_{\lambda} \delta_{\lambda \mu}$.

\section{Products of CHARACTER BASES}

In [23] we showed that the structure coefficients for the character basis $\tilde{s}_{\lambda}$ are the reduced (or stable) Kronecker coefficients, $\bar{g}_{\alpha, \beta}^{\gamma}$,

$$
\tilde{s}_{\alpha} \tilde{s}_{\beta}=\sum_{\gamma} \bar{g}_{\alpha, \beta}^{\gamma} \tilde{s}_{\gamma} .
$$

One of the main motivations for introducing these bases and developing their properties is that it will hopefully lead to a combinatorial interpretation for these coefficients.

In [24] we studied several combinatorial formulae for coefficients of repeated products of character bases in terms of multiset tableaux satisfying a lattice condition. In particular, we gave a combinatorial interpretation for the coefficient of $\tilde{s}_{\nu}$ in products of the form

$$
\tilde{s}_{\lambda_{1}} \tilde{s}_{\lambda_{2}} \cdots \tilde{s}_{\lambda_{r}} \tilde{s}_{\gamma}
$$

where $\gamma$ is any partition and $\lambda_{i}$ 's are positive integers. Notice that these products contain the Pieri rule as a special case. Unfortunately, we do not have the means to extend this to a combinatorial interpretation for the coefficients $\bar{g}_{\alpha, \beta}^{\gamma}$. In order to gain a better understanding we develop product formulae for other bases in this section and coproduct formulae in Section 4.

3.1. Products on THE POWER SUM BASES. In contrast with the power symmetric function $p_{\lambda}$ that is multiplicative, the new bases $\left\{\mathbf{p}_{\gamma}\right\}$ and $\left\{\overline{\mathbf{p}}_{\gamma}\right\}$ are not multiplicative unless the parts of the partition are disjoint. Therefore, in this section we begin by describing the structure of products of the form $\mathbf{p}_{a^{b}} \mathbf{p}_{a^{c}}$ and $\overline{\mathbf{p}}_{a^{b}} \overline{\mathbf{p}}_{a^{c}}$.

Recall that the $k$-falling factorial, $(x)_{k}$, has the following product structure

$$
(x)_{n}(x)_{m}=\sum_{k \geqslant 0}\left(\begin{array}{l}
m \\
k
\end{array}\right)\left(\begin{array}{l}
n \\
k
\end{array}\right) k !(x)_{m+n-k} .
$$

Therefore we can at least easily compute the following product of one of the power sum bases from this formula.

Proposition 3.1. For a positive integers $i, r$ and $s$,

$$
\overline{\mathbf{p}}_{i^{r}} \overline{\mathbf{p}}_{i^{s}}=\sum_{k \geqslant 0}\left(\begin{array}{l}
r \\
k
\end{array}\right)\left(\begin{array}{l}
s \\
k
\end{array}\right) k ! i^{k} \overline{\mathbf{p}}_{i^{r+s-k}} .
$$

If $m_{i}(\lambda)=0$, then $\overline{\mathbf{p}}_{i^{r}} \overline{\mathbf{p}}_{\lambda}=\overline{\mathbf{p}}_{\left(i^{r}\right) \cup \lambda}$. 
It also implies by making a change of basis back and forth between $\mathbf{p}_{i^{r}}$ to $\overline{\mathbf{p}}_{i^{s}}$ that we have the following expansion for the product of the $\mathbf{p}_{i^{r}}$ elements.

Proposition 3.2. Define the coefficients

$$
c_{i, r, s, a}=\sum_{\ell \geqslant 0} \sum_{d \geqslant 0} \sum_{k \geqslant 0}(-1)^{r+s-\ell-d}\left(\begin{array}{l}
r \\
\ell
\end{array}\right)\left(\begin{array}{l}
s \\
d
\end{array}\right)\left(\begin{array}{l}
\ell \\
k
\end{array}\right)\left(\begin{array}{l}
d \\
k
\end{array}\right)\left(\begin{array}{c}
\ell+d-k \\
a
\end{array}\right) k ! i^{k}
$$

then

$$
\mathbf{p}_{i^{r}} \mathbf{p}_{i^{s}}=\sum_{a=0}^{r+s} c_{i, r, s, a} \mathbf{p}_{i^{a}} .
$$

If $m_{i}(\lambda)=0$, then $\mathbf{p}_{i^{r}} \mathbf{p}_{\lambda}=\mathbf{p}_{\left(i^{r}\right) \cup \lambda}$.

3.2. Products of induced trivial Characters. A combinatorial interpretation for the Kronecker product of two complete symmetric functions expanded in the complete basis is listed as Exercise 23 (e) in section I.7 of [18] and Exercise 7.84 (b) in [30]. The earliest reference to this result that we are aware of is due to Garsia and Remmel [12]. The formula for this Kronecker product is

$$
h_{\lambda} * h_{\mu}=\sum_{M} \prod_{i=1}^{\ell(\lambda)} \prod_{j=1}^{\ell(\mu)} h_{M_{i j}}
$$

summed over all matrices $M$ of non-negative integers with $\ell(\lambda)$ rows, $\ell(\mu)$ columns and row sums are given by the vectors $\lambda$ and column sums by the vector $\mu$. For $\lambda, \mu, \nu \vdash n$, we define $d_{\lambda \mu \nu}$ as the coefficient of $h_{\nu}$ in $h_{\lambda} * h_{\mu}$.

Let $\lambda, \mu$ and $\nu$ be partitions, then for $n \geqslant|\lambda|+|\mu|+|\nu|$, let $\bar{d}_{\lambda \mu \nu}:=$ $d_{(n-|\lambda|, \lambda)(n-|\mu|, \mu)(n-|\nu|, \nu)}$ denote the common (stable) coefficient of $h_{(n-|\nu|, \nu)}$ in $h_{(n-|\lambda|, \lambda)} * h_{(n-|\mu|, \mu)}$.

Proposition 3.3. For partitions $\lambda$ and $\mu$

$$
\tilde{h}_{\lambda} \tilde{h}_{\mu}=\sum_{\nu:|\nu| \leqslant|\lambda|+|\mu|} \bar{d}_{\lambda \mu \nu} \tilde{h}_{\nu}
$$

Proof. For each partition $\gamma$ such that $|\gamma| \geqslant n$ ( $n$ sufficiently large), calculation in terms of characters shows that

$$
\begin{aligned}
\tilde{h}_{\lambda}\left[\Xi_{\gamma}\right] \tilde{h}_{\mu}\left[\Xi_{\gamma}\right] & =\left\langle h_{|\gamma|-|\lambda|} h_{\lambda}, p_{\gamma}\right\rangle\left\langle h_{|\gamma|-|\mu|} h_{\mu}, p_{\gamma}\right\rangle \\
& =\left\langle h_{(|\gamma|-|\lambda|, \lambda)} * h_{(|\gamma|-|\mu|, \mu)}, p_{\gamma}\right\rangle \\
& =\sum_{|\nu| \leqslant|\lambda|+|\mu|} \bar{d}_{\lambda \mu \nu}\left\langle h_{(|\gamma|-|\nu|, \nu)}, p_{\gamma}\right\rangle \\
& =\sum_{|\nu| \leqslant|\lambda|+|\mu|} \bar{d}_{\lambda \mu \nu} \tilde{h}_{\nu}\left[\Xi_{\gamma}\right] .
\end{aligned}
$$

We can conclude by Proposition 2.1 that the structure coefficients of the induced trivial characters are the coefficients $\bar{d}_{\lambda \mu \nu}$.

Let $S$ be a multiset and $T$ a set. The restriction of $S$ to $T$ is the multiset $\left.S\right|_{T}=$ $\{\{v \in S: v \in T\}$. Then we can define the restriction of a multiset partition to the content $T$ by $\left.\pi\right|_{T}=\left\{\left\{\left.S\right|_{T}: S \in \pi\right\}\right.$. If necessary in this operation we throw away empty multisets in $\left.\pi\right|_{T}$.

We will use the notation $\pi \# \tau$ to represent a set of multiset partitions that will appear in the product. Let $\pi$ and $\tau$ be multiset partitions on disjoint sets $S$ and $T$.

$$
\pi \# \tau=\left\{\theta: \theta H S \cup T,\left.\theta\right|_{S}=\pi,\left.\theta\right|_{T}=\tau\right\} .
$$


That is, $\theta \in \pi \# \tau$ means that $\theta$ is of the form

$$
\theta=\left\{\left\{S_{i_{1}}, \ldots, S_{i_{\ell(\pi)-k}}, T_{j_{1}}, \ldots, T_{j_{\ell(\tau)-k}}, S_{i_{1}^{\prime}} \cup T_{j_{1}^{\prime}}, \ldots, S_{i_{k}^{\prime}} \cup T_{j_{k}^{\prime}}\right\}\right.
$$

where

and

$$
\left\{i_{1}, i_{2}, \ldots, i_{\ell(\pi)-k}, i_{1}^{\prime}, i_{2}^{\prime}, \ldots, i_{k}^{\prime}\right\}=\{1,2, \ldots, \ell(\pi)\}
$$

$$
\left\{j_{1}, j_{2}, \ldots, j_{\ell(\tau)-k}, j_{1}^{\prime}, j_{2}^{\prime}, \ldots, j_{k}^{\prime}\right\}=\{1,2, \ldots, \ell(\tau)\}
$$

We propose the following different, but equivalent combinatorial interpretation for this product of the induced trivial character basis.

Proposition 3.4. For multiset partitions $\pi+S$ and $\tau+T$ where the multisets $S$ and T are disjoint,

$$
\tilde{h}_{\tilde{m}(\pi)} \tilde{h}_{\tilde{m}(\tau)}=\sum_{\theta \in \pi \# \tau} \tilde{h}_{\tilde{m}(\theta)} .
$$

Before we give a proof of this proposition by showing an equivalence with Equation (13), we provide an example to try to clarify any subtleties of the notation.

EXAMPLE 3.5. Let $\pi=\{\{\{1\}\},\{\{1\}\},\{\{2\}\}\}$ and $\tau=\{\{\{3\}\},\{\{3\}\},\{\{4\}\}\}$. Below we list the multiset partitions in $\pi \# \tau$ along with the corresponding partition $\tilde{m}(\theta)$.

$$
\begin{aligned}
& \{\{\{1\},\{\{1\},\{\{2\},\{\{3\},\{\{3\},\{\{4\}\}\} \rightarrow 2211 \quad\{\{\{1,3\}\},\{1\}\},\{\{2\},\{\{3\},\{\{4\}\}\} \rightarrow 11111 \\
& \{\{\{1\},\{\{1\},\{\{2,3\},\{\{3\},\{\{4\}\} \rightarrow 2111\{\{\{1\}\},\{11,4\},\{\{2\},\{\{3\},\{\{3\}\}\} \rightarrow 2111 \\
& \{\{\{1\},\{\{1\},\{\{2,4\},\{\{3\},\{\{3\}\}\} \rightarrow 221\}\{\{\{1,3\},,\{1,3\}\},\{\{2\},,\{4\}\}\} \rightarrow 211 \\
& \{\{\{1,3\},\{\{1,4\}\},\{\{2\},\{\{3\}\}\} \rightarrow 1111 \quad\{\{\{1\},\{\{1,3\}\},\{\{2,3\}\},\{\{4\}\}\} \rightarrow 1111 \\
& \{\{\{1\},\{\{1,4\},\{\{2,3\},\{\{3\}\}\} \rightarrow 1111 \quad\{\{\{1\},\{\{1,3\}\},\{\{2,4\},\{\{3\}\}\} \rightarrow 1111 \\
& \{\{\{1,3\},\{\{1,3\},\{\{2,4\}\}\} \rightarrow 21 \quad\{\{\{1,3\},\{\{1,4\},\{\{2,3\}\}\} \rightarrow 111
\end{aligned}
$$

As a consequence of Proposition 3.4 we conclude

$$
\tilde{h}_{21} \tilde{h}_{21}=\tilde{h}_{111}+4 \tilde{h}_{1111}+\tilde{h}_{11111}+\tilde{h}_{21}+\tilde{h}_{211}+2 \tilde{h}_{2111}+\tilde{h}_{221}+\tilde{h}_{2211}
$$

or in terms of Kronecker products with $n=8$,

$$
h_{521} * h_{521}=h_{5111}+4 h_{41111}+h_{311111}+h_{521}+h_{4211}+2 h_{32111}+h_{3221}+h_{22211} .
$$

Proof. We define a bijection between matrices whose row sums are $(n-|\lambda|, \lambda)$ and whose column sums are $(n-|\mu|, \mu)$ and elements of $\pi \# \tau$ where $\pi$ is a multiset partition such that $\tilde{m}(\pi)$ is $\lambda$ and $\tau$ is a multiset partition such that $\tilde{m}(\tau)=\mu$.

Let $M$ be such a matrix. The first row of this matrix has sum equal to $n-|\lambda|$ and the sum of row $i$ of this matrix represents the number of times that some multiset $A$ repeats in $\pi$ (it does not matter what that multiset is, just that it repeats $\sum_{j} M_{i j}$ times). The sum of column $j$ of this matrix (for $j>1$ ) represents the number of times that a particular part of the multiset $B$ repeats in $\tau$ (again, it does not matter the content of that multiset, just that it is different than the others). Therefore the entry $M_{i j}$ is the number of times that $A \cup B$ repeats in the multiset $\theta \in \pi \# \tau$. The value of $M_{i 1}$ is equal to the number of times that $A$ appears in $\theta$ and the value of $M_{1 j}$ is the number of times that $B$ appears in $\theta$.

EXAMPLE 3.6. To ensure that the bijection described in the proof is clear we show the correspondence between some specific multiset partitions and the non-negative integer matrices to which they correspond. The second and third row will represent the multiplicities of $\{\{1\}$ and $\{2\}$ respectively. The second and third column will represent the multiplicities of $\{\{3\}\}$ and $\{\{4\}$ respectively. Rather than consider all 
multiset partitions, we will consider only the 4 that we calculated in the last example that have $\tilde{m}(\pi)=1111$.

$$
\begin{aligned}
& \left\{\{\{1,3\}\},\{\{1,4\}\},\{\{2\},\{\{3\}\}\} \leftrightarrow\left[\begin{array}{rrr}
n-4 & 1 & 0 \\
0 & 1 & 1 \\
1 & 0 & 0
\end{array}\right]\right. \\
& \left\{\left\{\{1\},\{\{1,3\}\},\{\{2,3\},\{\{4\}\}\} \leftrightarrow\left[\begin{array}{rrr}
n-4 & 0 & 1 \\
1 & 1 & 0 \\
0 & 1 & 0
\end{array}\right]\right.\right. \\
& \left\{\left\{\{1\},\{\{1,4\}\},\{\{2,3\},\{\{3\}\}\} \leftrightarrow\left[\begin{array}{rrr}
n-4 & 1 & 0 \\
1 & 0 & 1 \\
0 & 1 & 0
\end{array}\right]\right.\right. \\
& \left\{\left\{\{1\},\left\{\{1,3\},\{\{2,4\},\{\{3\}\}\} \leftrightarrow\left[\begin{array}{ccc}
n-4 & 1 & 0 \\
1 & 1 & 0 \\
0 & 0 & 1
\end{array}\right]\right.\right.\right.
\end{aligned}
$$

The multiset partition notation is therefore not significantly different than the integer matrices notation, but there are distinct advantages to an interpretation in terms of multiset partitions. The main one is that the notion of multisets in the context of symmetric functions leads to the combinatorial objects of multiset tableaux that can be used as a possible object to keep track of stable Kronecker coefficients.

\section{Coproducts of the character bases}

The coproduct of symmetric functions corresponds to restriction from $S_{n}$ to $S_{r} \times$ $S_{t}$ where $r+t=n$. This is because the coproduct operation is isomorphic to the operation of replacing one set of variables with two in the power sum symmetric function $p_{k}[X] \rightarrow p_{k}[X]+p_{k}[Y]$ and the evaluation of the symmetric function at the eigenvalues of $S_{r} \times S_{t}$ in $S_{n}$ replaces the $X$ variables by eigenvalues of the element of $S_{r}$ and the $Y$ variables by the eigenvalues of the element of $S_{t}$. Therefore, formulae involving coproducts of character basis compute multiplicities for these restrictions. The main result are coproduct formulae for the analogues of the power sum bases, the $\tilde{h}, \tilde{s}$ and $\tilde{x}$ bases.

For a basis $\left\{b_{\lambda}\right\}$ of the symmetric functions, we will refer to the coproduct structure coefficients as the coefficients of $b_{\mu} \otimes b_{\nu}$ in $\Delta\left(b_{\lambda}\right)$ where $\lambda, \mu, \nu$ are all partitions. For bases that are of homogeneous degree we have the restriction that $|\mu|+|\nu|=|\lambda|$, but for the bases here it could be the case that $|\mu|+|\nu| \leqslant|\lambda|$. To summarize the results in this section we state the following theorem.

THEOREM 4.1. The coproduct formulae for the character bases are given by:

- (Theorem 4.7) For partitions $\lambda, \mu$ and $\nu$ with $|\mu|+|\nu| \leqslant|\lambda|$, the coproduct structure coefficients of the basis $\left\{\tilde{s}_{\lambda}\right\}$ are $\sum_{\gamma} c_{\mu \gamma}^{\lambda}$ where the sum is over all partitions $\gamma$ such that $\gamma / \nu$ is a horizontal strip of size $|\lambda|-|\mu|-|\nu|$ and $c_{\mu \gamma}^{\lambda}$ are the Littlewood-Richardson coefficients.

- (Propositions 4.3, 4.5 and Corollary 4.6 respectively) The bases $\left\{\overline{\mathbf{p}}_{\lambda}\right\},\left\{\tilde{h}_{\lambda}\right\}$ and $\left\{\tilde{x}_{\lambda}\right\}$ have the same coproduct structure coefficients as the power sum $\left\{p_{\lambda}\right\}$, complete $\left\{h_{\lambda}\right\}$ and Schur bases $\left\{s_{\lambda}\right\}$ (respectively).

- (Proposition 4.4) For the basis $\left\{\mathbf{p}_{\lambda}\right\}$, the second analogue of the power sum basis, we have $\Delta\left(\mathbf{p}_{\gamma}\right):=\prod_{i \geqslant 1} \Delta\left(\mathbf{p}_{i^{m_{i}(\gamma)}}\right)$ where

$$
\Delta\left(\mathbf{p}_{i^{r}}\right)=\sum_{d=0}^{r}\left(\begin{array}{l}
r \\
d
\end{array}\right) \sum_{a=0}^{d}\left(\begin{array}{l}
d \\
a
\end{array}\right) \mathbf{p}_{i^{a}} \otimes \mathbf{p}_{i^{d-a}} .
$$


4.1. Coproducts ON THE POWER SUM BASES. In [23] we derived an analogue of the Murnaghan-Nakayama rule, see Theorem 20. This allows us to expand the power symmetric function in the irreducible character basis. This is currently the most efficient method for computing the irreducible character basis.

For a partition $\lambda$, it follows from the defining relations in Equation (4) that

$$
\overline{\mathbf{p}}_{\lambda}=\sum_{\mu \vdash|\lambda|}\left\langle p_{\lambda}, s_{\mu}\right\rangle \tilde{x}_{\mu}=\sum_{\mu \vdash|\lambda|}\left\langle p_{\lambda}, m_{\mu}\right\rangle \tilde{h}_{\mu} .
$$

Now this power sum analogue is important because of the following proposition.

Proposition 4.2. If $|\mu|<|\lambda|$, then $\overline{\mathbf{p}}_{\lambda}\left[\Xi_{\mu}\right]=0$ and if $|\mu|=|\lambda|$, then $\overline{\mathbf{p}}_{\lambda}\left[\Xi_{\mu}\right]=\delta_{\lambda \mu}$. More generally, $\phi_{m}\left(\overline{\mathbf{p}}_{\lambda}\right)=h_{(m-|\lambda|)} p_{\lambda}$.

Proof. We know that $\tilde{h}_{\lambda}\left[\Xi_{\gamma}\right]=\left\langle h_{(|\gamma|-|\mu|)} h_{\mu}, p_{\gamma}\right\rangle$ (and in particular the expression is 0 if $|\gamma|<|\mu|)$ by Equation (6) of [23]. By Equation (15),

$$
\begin{aligned}
\phi_{m}\left(\overline{\mathbf{p}}_{\lambda}\right) & =\sum_{\gamma \vdash m} \sum_{\mu \vdash|\lambda|}\left\langle p_{\lambda}, m_{\mu}\right\rangle \tilde{h}_{\mu}\left[\Xi_{\gamma}\right] \frac{p_{\gamma}}{z_{\gamma}} \\
& =\sum_{\gamma \vdash m} \sum_{\mu \vdash|\lambda|}\left\langle p_{\lambda}, m_{\mu}\right\rangle\left\langle h_{(m-|\mu|)} h_{\mu}, p_{\gamma}\right\rangle \frac{p_{\gamma}}{z_{\gamma}}=\sum_{\gamma \vdash m}\left\langle h_{(m-|\mu|)} p_{\lambda}, p_{\gamma}\right\rangle \frac{p_{\gamma}}{z_{\gamma}}
\end{aligned}
$$

by an application of Equation (2) and the right hand side of this expression is equal to $h_{(m-|\mu|)} p_{\lambda}$ by a second application of Equation (2).

We wish to understand as completely as possible the coproduct structure on these power sum bases. Since the coproduct is an algebra homomorphism, it suffices to understand the result on the partitions of the form $\left(i^{r}\right)$.

Proposition 4.3. The $\overline{\mathbf{p}}_{\lambda}$ basis has the same coproduct as the power sum basis. That is, if the coefficients $a_{\gamma \tau}^{\mu}$ are defined by those appearing in the equation $\Delta\left(p_{\mu}\right)=$ $\sum_{\gamma, \tau} a_{\gamma \tau}^{\mu} p_{\gamma} \otimes p_{\tau}$ then $\Delta\left(\overline{\mathbf{p}}_{\mu}\right)=\sum_{\gamma, \tau} a_{\gamma \tau}^{\mu} \overline{\mathbf{p}}_{\gamma} \otimes \overline{\mathbf{p}}_{\tau}$ and specifically

$$
\Delta\left(\overline{\mathbf{p}}_{i^{r}}\right)=\sum_{k=0}^{r}\left(\begin{array}{l}
r \\
k
\end{array}\right) \overline{\mathbf{p}}_{i^{k}} \otimes \overline{\mathbf{p}}_{i^{r-k}} .
$$

Proof. Define the coefficients $b_{\alpha \beta}^{\lambda}$ appearing in the coproduct of the complete symmetric function basis by

$$
\Delta\left(h_{\lambda}\right)=\sum_{\alpha, \beta} b_{\alpha \beta}^{\lambda} h_{\alpha} \otimes h_{\beta} .
$$

In Proposition 4.5, we will show by direct computation that $\Delta\left(\tilde{h}_{\lambda}\right)=\sum_{\alpha, \beta} b_{\alpha \beta}^{\lambda} \tilde{h}_{\alpha} \otimes \tilde{h}_{\beta}$. We know already that the coefficient of $p_{\gamma}$ in $h_{\lambda}$ is equal to the coefficient of $\overline{\mathbf{p}}_{\gamma}$ in $\tilde{h}_{\lambda}$ (and both are equal to $\left\langle h_{\lambda}, p_{\gamma} / z_{\gamma}\right\rangle$ by Equation (4)). Therefore the coefficient of $\overline{\mathbf{p}}_{\gamma} \otimes \overline{\mathbf{p}}_{\tau}$ in $\Delta\left(\overline{\mathbf{p}}_{\lambda}\right)$ is equal to

$$
\begin{aligned}
\Delta\left(\overline{\mathbf{p}}_{\lambda}\right) & =\sum_{\nu}\left\langle p_{\lambda}, m_{\nu}\right\rangle \Delta\left(\tilde{h}_{\nu}\right)=\sum_{\nu} \sum_{\alpha, \beta}\left\langle p_{\lambda}, m_{\nu}\right\rangle b_{\alpha \beta}^{\nu} \tilde{h}_{\alpha} \otimes \tilde{h}_{\beta} \\
& =\sum_{\nu} \sum_{\alpha, \beta} \sum_{\gamma, \tau}\left\langle p_{\lambda}, m_{\nu}\right\rangle b_{\alpha \beta}^{\nu}\left\langle h_{\alpha}, p_{\gamma}\right\rangle\left\langle h_{\beta}, p_{\tau}\right\rangle \frac{\overline{\mathbf{p}}_{\gamma}}{z_{\gamma}} \otimes \frac{\overline{\mathbf{p}}_{\tau}}{z_{\tau}} .
\end{aligned}
$$

Now if we compute the coefficient of $p_{\gamma} \otimes p_{\tau}$ in $\Delta\left(p_{\lambda}\right)$ it is precisely the same as the coefficient of $\overline{\mathbf{p}}_{\gamma} \otimes \overline{\mathbf{p}}_{\tau}$ in $\Delta\left(\overline{\mathbf{p}}_{\lambda}\right)$.

In particular, Equation (16) holds because

$$
\Delta\left(p_{i^{r}}\right)=\left(p_{i} \otimes 1+1 \otimes p_{i}\right)^{r}=\sum_{k=0}^{r}\left(\begin{array}{l}
r \\
k
\end{array}\right) p_{i^{k}} \otimes p_{i^{r-k}}
$$


Now for the coproduct on the other power basis $\mathbf{p}_{\lambda}$ we need to work slightly harder to give the expression.

Proposition 4.4. For $i, r \geqslant 1$, the coproduct on $\mathbf{p}_{\lambda}$ can be calculated by

$$
\Delta\left(\mathbf{p}_{i^{r}}\right)=\sum_{d=0}^{r}\left(\begin{array}{l}
r \\
d
\end{array}\right) \sum_{a=0}^{d}\left(\begin{array}{l}
d \\
a
\end{array}\right) \mathbf{p}_{i^{a}} \otimes \mathbf{p}_{i^{d-a}},
$$

and since $\Delta$ is an algebra homomorphism, $\Delta\left(\mathbf{p}_{\gamma}\right):=\prod_{i \geqslant 1} \Delta\left(\mathbf{p}_{i^{m_{i}(\gamma)}}\right)$.

Proof. Applying Equation (3) and (16), we simplify the limits of the expression to show that $\left(\begin{array}{l}r \\ k\end{array}\right)=0$ if $k>r$. Then we see that

$$
\begin{aligned}
\Delta\left(\mathbf{p}_{i^{r}}\right) & =\sum_{k \geqslant 0}(-1)^{r-k}\left(\begin{array}{l}
r \\
k
\end{array}\right) \Delta\left(\overline{\mathbf{p}}_{i^{k}}\right) \\
& =\sum_{k \geqslant 0} \sum_{\ell \geqslant 0}(-1)^{r-k}\left(\begin{array}{l}
r \\
k
\end{array}\right)\left(\begin{array}{l}
k \\
\ell
\end{array}\right) \overline{\mathbf{p}}_{i^{\ell}} \otimes \overline{\mathbf{p}}_{i^{k-\ell}} \\
& =\sum_{k \geqslant 0} \sum_{\ell \geqslant 0} \sum_{a \geqslant 0} \sum_{b \geqslant 0}(-1)^{r-k}\left(\begin{array}{l}
r \\
k
\end{array}\right)\left(\begin{array}{l}
k \\
\ell
\end{array}\right)\left(\begin{array}{c}
\ell \\
a
\end{array}\right)\left(\begin{array}{c}
k-\ell \\
b
\end{array}\right) \mathbf{p}_{i^{a}} \otimes \mathbf{p}_{i^{b}} \\
& =\sum_{a \geqslant 0} \sum_{b \geqslant 0} \sum_{k \geqslant 0} \sum_{\ell \geqslant 0}(-1)^{r-k}\left(\begin{array}{l}
r \\
k
\end{array}\right)\left(\begin{array}{c}
k \\
\ell
\end{array}\right)\left(\begin{array}{c}
\ell \\
a
\end{array}\right)\left(\begin{array}{c}
k-\ell \\
b
\end{array}\right) \mathbf{p}_{i^{a}} \otimes \mathbf{p}_{i^{b}}
\end{aligned}
$$

where in the last equality we have just rearranged the order of the summations. The sum over $a$ and $b$ can be combined by setting $d=a+b$ and changing the sum over $a \geqslant 0, b \geqslant 0$ to one of $d \geqslant 0, a \geqslant 0$. Moreover, since $\left(\begin{array}{l}\ell \\ a\end{array}\right)=0$ if $\ell<a$, then we can assume that $\ell \geqslant a$ and hence

$$
\begin{aligned}
\Delta\left(\mathbf{p}_{i^{r}}\right) & =\sum_{d \geqslant 0} \sum_{a=0}^{d} \sum_{k \geqslant 0} \sum_{\ell \geqslant a}(-1)^{r-k}\left(\begin{array}{l}
r \\
k
\end{array}\right)\left(\begin{array}{l}
k \\
\ell
\end{array}\right)\left(\begin{array}{l}
\ell \\
a
\end{array}\right)\left(\begin{array}{c}
k-\ell \\
d-a
\end{array}\right) \mathbf{p}_{i^{a}} \otimes \mathbf{p}_{i^{d-a}} \\
& =\sum_{d \geqslant 0} \sum_{a=0}^{d} \sum_{k \geqslant 0} \sum_{\ell \geqslant 0}(-1)^{r-k}\left(\begin{array}{l}
r \\
k
\end{array}\right)\left(\begin{array}{c}
k \\
\ell+a
\end{array}\right)\left(\begin{array}{c}
\ell+a \\
a
\end{array}\right)\left(\begin{array}{c}
k-\ell-a \\
d-a
\end{array}\right) \mathbf{p}_{i^{a}} \otimes \mathbf{p}_{i^{d-a}} .
\end{aligned}
$$

Now by expanding the binomial coefficients we know that

$$
\left(\begin{array}{c}
k \\
\ell+a
\end{array}\right)\left(\begin{array}{c}
\ell+a \\
a
\end{array}\right)\left(\begin{array}{c}
k-\ell-a \\
d-a
\end{array}\right)=\left(\begin{array}{l}
d \\
a
\end{array}\right)\left(\begin{array}{c}
k \\
\ell+d
\end{array}\right)\left(\begin{array}{c}
\ell+d \\
d
\end{array}\right) .
$$

Note that by taking the coefficient of $y^{d}$ in

$$
\begin{aligned}
\sum_{m=0}^{k} 2^{k-m}\left(\begin{array}{c}
k \\
m
\end{array}\right) y^{m} & =(1+1+y)^{k}=\sum_{m \geqslant 0} \sum_{\ell \geqslant 0}\left(\begin{array}{c}
k \\
\ell
\end{array}\right)\left(\begin{array}{c}
\ell \\
m
\end{array}\right) y^{m} \\
& =\sum_{m \geqslant 0} \sum_{\ell^{\prime} \geqslant 0}\left(\begin{array}{c}
k \\
\ell^{\prime}+m
\end{array}\right)\left(\begin{array}{c}
\ell^{\prime}+m \\
m
\end{array}\right) y^{m}
\end{aligned}
$$

we know that

$$
2^{k-d}\left(\begin{array}{l}
k \\
d
\end{array}\right)=\sum_{\ell \geqslant 0}\left(\begin{array}{c}
k \\
\ell+d
\end{array}\right)\left(\begin{array}{c}
\ell+d \\
d
\end{array}\right)
$$


This reduces Equation (18) to

$$
\begin{aligned}
\Delta\left(\mathbf{p}_{i^{r}}\right) & =\sum_{d \geqslant 0} \sum_{a=0}^{d}\left(\begin{array}{l}
d \\
a
\end{array}\right) \sum_{k \geqslant 0}(-1)^{r-k} 2^{k-d}\left(\begin{array}{l}
r \\
k
\end{array}\right)\left(\begin{array}{l}
k \\
d
\end{array}\right) \mathbf{p}_{i^{a}} \otimes \mathbf{p}_{i^{d-a}} \\
& =\sum_{d \geqslant 0} \sum_{a=0}^{d}\left(\begin{array}{l}
d \\
a
\end{array}\right)\left(\begin{array}{l}
r \\
d
\end{array}\right) \mathbf{p}_{i^{a}} \otimes \mathbf{p}_{i^{d-a}}
\end{aligned}
$$

The last equality follows by taking the coefficient of $y^{d}$ in

$$
\sum_{m \geqslant 0}\left(\begin{array}{c}
r \\
m
\end{array}\right) y^{m}=(2-1+y)^{r}=\sum_{m \geqslant 0} \sum_{k \geqslant 0}(-1)^{r-k} 2^{k-m}\left(\begin{array}{l}
r \\
k
\end{array}\right)\left(\begin{array}{c}
k \\
m
\end{array}\right) y^{m} .
$$

4.2. Coproducts ON THE INDUCED TRIVIAL AND INDUCED IRREDUCIBLE CHARACTER BASIS. The induced trivial character basis follows the combinatorics of multiset partitions for the product. We now give the coproduct for this basis.

It turns out that the coefficient of $\tilde{h}_{\mu} \otimes \tilde{h}_{\nu}$ in $\Delta\left(\tilde{h}_{\lambda}\right)$ is equal to the coefficient $h_{\mu} \otimes h_{\nu}$ in $\Delta\left(h_{\lambda}\right)$. Since $\Delta\left(h_{n}\right)=\sum_{k=0}^{n} h_{n-k} \otimes h_{k}$, we have more generally that

$$
\Delta\left(h_{\lambda}\right)=\sum_{\alpha+\beta=\lambda} h_{\alpha} \otimes h_{\beta}
$$

Hence the coproduct formula for the induced trivial character basis can be stated in the following proposition.

Proposition 4.5. For a partition $\lambda$,

$$
\Delta\left(\tilde{h}_{\lambda}\right)=\sum_{\alpha+\beta=\lambda} \tilde{h}_{\alpha} \otimes \tilde{h}_{\beta}
$$

where the sum is over all pairs of weak compositions $\alpha$ and $\beta$ of length $\lambda$ whose vector sum is equal to $\lambda$.

Proof. We will show that $\tilde{h}_{\lambda}[X+Y]=\sum_{\alpha+\beta=\lambda} \tilde{h}_{\alpha}[X] \tilde{h}_{\beta}[Y]$. This will be done by applying Proposition 2.1 that states if $f\left[\Xi_{\mu}\right]=g\left[\Xi_{\mu}\right]$ for enough partitions $\mu$, then $f=g$ as symmetric functions.

We note that for partitions $\mu$ and $\nu$ and a positive integer $n=|\mu|+|\nu|$,

$$
\tilde{h}_{\lambda}\left[\Xi_{\mu}+\Xi_{\nu}\right]=\left\langle h_{(n-|\lambda|, \lambda)}, p_{\mu} p_{\nu}\right\rangle .
$$

Now the evaluation of the,

$$
\begin{aligned}
\sum_{\alpha+\beta=\lambda} \tilde{h}_{\alpha}\left[\Xi_{\mu}\right] \tilde{h}_{\beta}\left[\Xi_{\nu}\right] & =\sum_{\alpha+\beta=\lambda}\left\langle h_{(|\mu|-|\alpha|, \alpha)}, p_{\mu}\right\rangle\left\langle h_{(|\nu|-|\beta|, \beta)}, p_{\nu}\right\rangle \\
& =\sum_{\alpha+\beta=\lambda} \sum_{k=0}^{n-|\lambda|}\left\langle h_{(n-|\lambda|-k, \alpha)}, p_{\mu}\right\rangle\left\langle h_{(k, \beta)}, p_{\nu}\right\rangle
\end{aligned}
$$

where in the last expression all of the terms in sum are assumed to be 0 unless $k=|\nu|-|\beta|$. In this case, $n-k-|\lambda|=|\mu|+|\nu|-(|\nu|-|\beta|)-|\lambda|=|\mu|-|\alpha|$. Now we can recognize the terms in the left entry of the scalar product as those that arise as the coproduct formula on the complete basis element $h_{(n-|\lambda|, \lambda)}$. If we define $\left\langle f \otimes f^{\prime}, g \otimes g^{\prime}\right\rangle=\langle f, g\rangle\left\langle f^{\prime}, g^{\prime}\right\rangle$ then we know (see for instance [18, I.5 p. 92 Example 
25]) that $\langle\Delta(f), g \otimes h\rangle=\langle f, g h\rangle$. Hence we have that Equation (20) is equivalent to

$$
\begin{aligned}
\sum_{\alpha+\beta=\lambda} \tilde{h}_{\alpha}\left[\Xi_{\mu}\right] \tilde{h}_{\beta}\left[\Xi_{\nu}\right] & =\left\langle\Delta\left(h_{(n-|\lambda|, \lambda)}\right), p_{\nu} \otimes p_{\mu}\right\rangle \\
& =\left\langle h_{(n-|\lambda|, \lambda)}, p_{\nu} p_{\mu}\right\rangle \\
& =\tilde{h}_{\lambda}\left[\Xi_{\mu}+\Xi_{\nu}\right] .
\end{aligned}
$$

Now from Proposition 2.1 we can conclude that $\tilde{h}_{\lambda}\left[X+\Xi_{\nu}\right]=\sum_{\alpha+\beta=\lambda} \tilde{h}_{\alpha}[X] \tilde{h}_{\beta}\left[\Xi_{\nu}\right]$ as a symmetric function identity and a second application allows us to conclude that $\tilde{h}_{\lambda}[X+Y]=\sum_{\alpha+\beta=\lambda} \tilde{h}_{\alpha}[X] \tilde{h}_{\beta}[Y]$.

Because the relationship between the $\tilde{h}_{\lambda}$ basis and the $\tilde{x}_{\lambda}$ basis is the same as the relationship between the $h_{\lambda}$ and $s_{\lambda}$ basis, we can conclude that the coproduct rule on the induced irreducible character basis will be the same as that for the Schur basis.

Corollary 4.6. For a partition $\lambda$,

$$
\Delta\left(\tilde{x}_{\lambda}\right)=\sum_{\mu, \nu} c_{\mu \nu}^{\lambda} \tilde{x}_{\mu} \otimes \tilde{x}_{\nu}
$$

where the coefficients $c_{\mu \nu}^{\lambda}$ are the Littlewood-Richardson coefficients, the same coproduct structure coefficients for the Schur basis.

4.3. Coproducts on the IRreducible Character BAsis. The method that we used in the last section to derive coproduct formula can also be used to derive the coproduct for the irreducible character basis. In this case though we reverse the expression and expand $\tilde{s}_{\lambda}[X+Y]$ evaluated at $X=\Xi_{\mu}$ and $Y=\Xi_{\nu}$.

THEOREM 4.7. For a partition $\lambda$,

$$
\Delta\left(\tilde{s}_{\lambda}\right)=\sum_{\delta:|\delta| \leqslant|\lambda|} \sum_{\beta \vdash|\lambda|-|\delta|} \sum_{\eta} c_{\delta \beta}^{\lambda} \tilde{s}_{\delta} \otimes \tilde{s}_{\eta}
$$

where $c_{\delta \beta}^{\lambda}$ is the Littlewood-Richardson coefficient and the inner sum is over partitions $\eta$ such that the skew partition $\beta / \eta$ is a horizontal strip.

In particular, the coefficient of $\tilde{s}_{\delta} \otimes \tilde{s}_{\eta}$ in $\Delta\left(\tilde{s}_{\lambda}\right)$ is equal to

$$
\sum_{\beta} c_{\delta \beta}^{\lambda}
$$

where the sum is over all partitions $\beta$ such that $\beta / \eta$ is a horizontal strip of size $|\lambda|-|\delta|-|\eta|$.

Proof. Assume that $\mu \vdash N$ where $N$ is "sufficiently large". We can without loss of generality assume that $N$ is larger than $2|\lambda|$ because we will apply Proposition 2.1. We need to show that the following identity holds for all partitions $\mu$, such that $|\mu|>n$ for some $n$ which is at least as large the degree of the symmetric function.

$$
\begin{aligned}
\tilde{s}_{\lambda}\left[\Xi_{\mu}+\Xi_{\nu}\right] & =\left\langle s_{(|\mu|+|\nu|-|\lambda|, \lambda)}, p_{\mu} p_{\nu}\right\rangle \\
& =\left\langle\Delta\left(s_{(|\mu|+|\nu|-|\lambda|, \lambda)}\right), p_{\mu} \otimes p_{\nu}\right\rangle \\
& =\sum_{\alpha \vdash|\mu|}\left\langle s_{\alpha}, p_{\mu}\right\rangle\left\langle s_{(|\mu|+|\nu|-|\lambda|, \lambda) / \alpha}, p_{\nu}\right\rangle .
\end{aligned}
$$

Now we know that $|\alpha|=|\mu|>2|\lambda|$, this implies that $(|\mu|+|\nu|-|\lambda|, \lambda) / \alpha$ is a skew partition where the first row is disconnected. The skew Schur function $s_{(|\mu|+|\nu|-|\lambda|, \lambda) / \alpha}$ is then equal to $s_{\lambda / \bar{\alpha}} \cdot s_{(|\mu|+|\nu|-|\lambda|-|\alpha|+|\bar{\alpha}|)}=s_{\lambda / \bar{\alpha}} \cdot s_{(|\nu|-|\lambda|+|\bar{\alpha}|)}$ where $\bar{\alpha}$ is the partition $\alpha$ with the first row removed. Since we know that $\alpha \vdash|\mu|$ then $\alpha$ is determined from 
$\bar{\alpha}$. In addition, $\bar{\alpha}$ is contained in $\lambda$ for $s_{\lambda / \bar{\alpha}}$ to be defined, thus $|\bar{\alpha}| \leqslant|\lambda|$. Therefore Equation (23) is equivalent to

$$
\begin{aligned}
\tilde{s}_{\lambda}\left[\Xi_{\mu}+\Xi_{\nu}\right] & =\sum_{\bar{\alpha}:|\bar{\alpha}| \leqslant|\lambda|}\left\langle s_{(|\mu|-|\bar{\alpha}|, \bar{\alpha})}, p_{\mu}\right\rangle\left\langle s_{\lambda / \bar{\alpha}} \cdot s_{(|\nu|-|\lambda|+|\bar{\alpha}|)}, p_{\nu}\right\rangle \\
& =\sum_{\bar{\alpha}:|\bar{\alpha}| \leqslant|\lambda|} \sum_{\beta \vdash|\lambda|-|\bar{\alpha}|} c_{\bar{\alpha} \beta}^{\lambda}\left\langle s_{\left.(|\mu|-|\bar{\alpha}|, \bar{\alpha}), p_{\mu}\right\rangle\left\langle s_{\beta} \cdot s_{(|\nu|-|\lambda|+|\bar{\alpha}|)}, p_{\nu}\right\rangle} \sum_{\bar{\alpha}:|\bar{\alpha}| \leqslant|\lambda|} \sum_{\beta \vdash|\lambda|-|\bar{\alpha}|} \sum_{\gamma} c_{\bar{\alpha} \beta}^{\lambda}\left\langle s_{(|\mu|-|\bar{\alpha}|, \bar{\alpha})}, p_{\mu}\right\rangle\left\langle s_{\gamma}, p_{\nu}\right\rangle\right.
\end{aligned}
$$

where the sum over $\gamma$ is of partitions such that $\gamma / \beta$ is a horizontal strip of size $|\nu|-|\lambda|+|\bar{\alpha}|$. But if $\gamma / \beta$ is a horizontal strip, then $\beta / \bar{\gamma}$ will also be a horizontal strip. Moreover we know that since $\gamma \vdash|\nu|$ and $\gamma$ is determined by $\bar{\gamma}$, then Equation (24) is equivalent to

$$
\begin{aligned}
\tilde{s}_{\lambda}\left[\Xi_{\mu}+\Xi_{\nu}\right] & =\sum_{\bar{\alpha}:|\bar{\alpha}| \leqslant|\lambda|} \sum_{\beta \vdash|\lambda|-|\bar{\alpha}|} \sum_{\bar{\gamma}} c_{\bar{\alpha} \beta}^{\lambda}\left\langle s_{(|\mu|-|\bar{\alpha}|, \bar{\alpha})}, p_{\mu}\right\rangle\left\langle s_{(|\nu|-|\bar{\gamma}|, \bar{\gamma})}, p_{\nu}\right\rangle \\
& =\sum_{\bar{\alpha}:|\bar{\alpha}| \leqslant|\lambda|} \sum_{\beta \vdash|\lambda|-|\bar{\alpha}|} \sum_{\bar{\gamma}} c_{\bar{\alpha} \beta}^{\lambda} \tilde{s}_{\bar{\alpha}}\left[\Xi_{\mu}\right] \tilde{s}_{\bar{\gamma}}\left[\Xi_{\nu}\right]
\end{aligned}
$$

where the inner sums are over partitions $\bar{\gamma}$ such that $\beta / \bar{\gamma}$ is a horizontal strip. Using the same argument that we did for the induced trivial character basis at the end of the proof of Proposition 4.5, we conclude that

$$
\Delta\left(\tilde{s}_{\lambda}\right)=\sum_{\bar{\alpha}} \sum_{\beta \vdash|\lambda|-|\bar{\alpha}|} \sum_{\bar{\gamma}} c_{\bar{\alpha} \beta}^{\lambda} \tilde{s}_{\bar{\alpha}} \otimes \tilde{s}_{\bar{\gamma}} .
$$

Equation (25) is equivalent to Equation (22) by setting $\delta=\bar{\alpha}$ and $\eta=\bar{\gamma}$.

REMARK 4.8. The antipode of a Hopf algebra is part of its defining structure. In the case of the symmetric functions, the antipode is an involution $S: \mathrm{Sym} \rightarrow$ Sym where $S\left(s_{\lambda}\right)=(-1)^{|\lambda|} s_{\lambda^{\prime}}$. The result of Assaf-Speyer [1] implies $(-1)^{|\lambda|} S\left(\tilde{s}_{\lambda}\right)$ will be Schur positive and hence this expression expands positively in the irreducible character basis. A better understanding of the transition coefficients between the Schur basis and the irreducible character basis could be used to give a more precise formula for this expression.

\section{EXPANSION OF THE ELEMENTARY SYMMETRIC FUNCTIONS IN THE IRREDUCIBLE CHARACTER BASIS}

In [23] we gave the expansion of the complete symmetric function $h_{\mu}$ in terms of the irreducible character basis $\tilde{s}_{\lambda}$. In this section we give the $\tilde{s}_{\lambda}$ expansion of an elementary symmetric function $e_{\mu}$. The proof is similar to the proof of the irreducible character expansion of a complete symmetric function, see [23] Theorem 9. We will start by proving the expansion in the irreducible character basis except that we will reserve some of the detailed combinatorial calculations for last. We assume that there is a total order on the sets that appear in set partitions and create tableaux to keep track of the terms in the symmetric function expansion. The order chosen does not matter, all the matters is that there is a total order, in the examples we have chosen lexicographic order. Let shape $(T)$ be a partition representing the shape of a tableau $T$ and we again use the overline notation on a partition to represent the partition with the first part removed, $\bar{\lambda}=\left(\lambda_{2}, \lambda_{3}, \ldots, \lambda_{\ell_{\lambda}}\right)$. 
THEOREM 5.1. For a partition $\mu$,

$$
e_{\lambda}=\sum_{T} \tilde{s} \overline{\operatorname{shape}(T)}
$$

where the sum is over tableaux that are of skew shape $\nu /\left(\nu_{2}\right)$ for some partition $\nu$ and that are weakly increasing in rows and columns with non-empty sets as labels of the tableaux (not multisets, that is no repeated values are allowed) such that the content of the tableau is $\left\{\left[1^{\lambda_{1}}, 2^{\lambda_{2}}, \ldots, \ell^{\lambda_{\ell}}\right\}\right.$. A set is allowed to appear multiple times in the same column if and only if the set has an odd number of entries. A set is allowed to appear multiple times in the same row if and only if the set has an even number of entries.

Proof. In Corollary 5.16 we will show that

$$
e_{\lambda}\left[\Xi_{\mu}\right]=\sum_{\pi \vdash\left\{11^{\lambda_{1}}, 2^{\lambda_{2}}, \ldots, \ell^{\left(\lambda_{\ell}\right)}\right\}}\left\langle h_{n} h_{\tilde{m}_{e}(\pi)} e_{\tilde{m}_{o}(\pi)}, p_{\mu}\right\rangle .
$$

where $\tilde{m}_{e}(\pi)$ (resp. $\tilde{m}_{o}(\pi)$ ) is the partition representing the multiplicities of the sets in $\pi$ with an even (resp. odd) number of elements (see Example 5.10).

For the rest of this proof, we assume that the reader is familiar with the Pieri rules, [30, Chapter 7, p. 339-340], which state $h_{r} s_{\lambda}$ is the sum of terms $s_{\mu}$ where the Young diagram for $\mu$ differs from the Young diagram of $\lambda$ by adding cells that may occur in the same row, but not in the same column of the diagram. Similarly, $e_{r} s_{\lambda}$ is the sum of terms $s_{\mu}$ where the Young diagram for $\mu$ differs from the Young diagram of $\lambda$ by adding cells that may occur in the same column, but not in the same row of the diagram.

Now order the occurrences of the generators in the product $h_{n} h_{\tilde{m}_{e}(\pi)} e_{\tilde{m}_{o}(\pi)}$ so that they are in the same order as the total order that is chosen for the sets that appear in the set partitions. Using a tableau to keep track of the terms in the resulting Schur expansion of the product we will have that

$$
h_{n} h_{\tilde{m}_{e}(\pi)} e_{\tilde{m}_{o}(\pi)}=\sum_{T} s_{\text {shape }(T)}
$$

where the sum is over tableaux that have $n$ blank cells in the first row and sets as labels in the rest of the tableau. Because we multiply by a generator $h_{r}$ if a set with an even number of entries occurs $r$ times, then those sets with an even number of elements can appear multiple times in the same row, but not in the same column of the tableau. Similarly, because we multiply by a generator $e_{r}$ if a set with an odd number of entries occurs $r$ times, then those sets with an odd number of elements can appear multiple times in the same column, but not in the same row of the tableau.

If the shape of the tableau is of skew shape $\nu /(n)$ with $n \geqslant \nu_{2}$, there are the same number of tableaux of skew-shape $\left(\nu_{2}+|\bar{\nu}|, \bar{\nu}\right) /\left(\nu_{2}\right)$ because there is a bijection by deleting blank cells in the first row.

Therefore we have

$$
e_{\lambda}\left[\Xi_{\mu}\right]=\sum_{\left.\pi \vdash\left\{11^{\lambda_{1}}, 2^{\lambda_{2}}, \ldots, \ell^{\lambda_{\ell}}\right\}\right\}} \sum_{T}\left\langle s_{\operatorname{shape}(T)}, p_{\mu}\right\rangle=\sum_{\left.\pi \vdash\left\{1^{\lambda_{1}}, 2^{\lambda_{2}}, \ldots, \ell^{\lambda_{\ell}}\right\}\right\}} \sum_{T} \tilde{s}_{\operatorname{shape}(T)}\left[\Xi_{\mu}\right]
$$

where the sum is over those tableau described in the statement of the proposition. Our proposition now follows from Proposition 2.1. 
EXAmple 5.2. To begin with a small example, consider the expansion of $e_{21}$. The following 11 tableaux follow the rules outlined in Theorem 5.1.

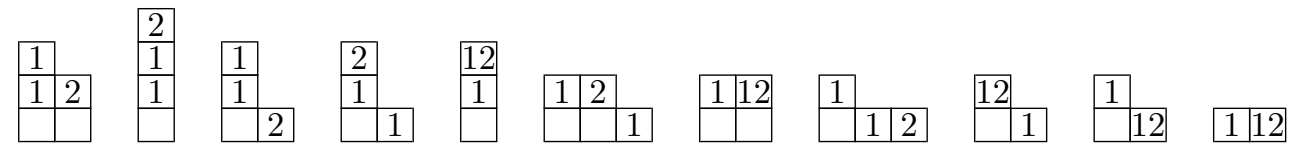

Theorem 5.1 then states that

$$
e_{21}=\tilde{s}_{21}+\tilde{s}_{111}+3 \tilde{s}_{11}+2 \tilde{s}_{2}+3 \tilde{s}_{1}+\tilde{s}_{()}
$$

EXAMPLE 5.3. A slightly larger example is the expansion of $e_{33}$. If we use Sage $[31,26]$ to determine the expansion we see that

$$
\begin{aligned}
e_{33}=2 \tilde{s}_{()}+4 \tilde{s}_{1}+4 \tilde{s}_{11}+4 \tilde{s}_{111}+4 \tilde{s}_{1111}+3 \tilde{s}_{11111} & +\tilde{s}_{111111}+6 \tilde{s}_{2} \\
+8 \tilde{s}_{21}+7 \tilde{s}_{211}+4 \tilde{s}_{2111}+\tilde{s}_{21111}+5 \tilde{s}_{22} & +4 \tilde{s}_{221}+\tilde{s}_{2211}+\tilde{s}_{222} \\
& +5 \tilde{s}_{3}+4 \tilde{s}_{31}+\tilde{s}_{311}+\tilde{s}_{32}+\tilde{s}_{4} .
\end{aligned}
$$

Hence there are 71 tableaux in total satisfying the conditions of Theorem 5.1 that have a total content $\left\{1^{3}, 2^{3}\right\}$. Listing all 71 tableaux is perhaps not a clear example so let us consider just the coefficient of $\tilde{s}_{21}$. Notice that the content of the tableaux is $\left\{1^{3}, 2^{3}\right\}$. Since the cells of the tableaux must contain sets, there are four ways to partition $\left\{\left\{1^{3}, 2^{3}\right\}\right.$ into sets: $\{\{\{1\},\{1\},\{1\},\{2\},\{2\},\{2\}\} ;\{\{\{1\},\{1\},\{1,2\},\{2\},\{2\}\}$; $\{\{\{1\},\{1,2\},\{1,2\},\{2\}\}\}$; and $\{\{\{1,2\},\{1,2\},\{1,2\}\}$. The shape of the tableaux is of the form $(r, 2,1) /(2)$ where $r$ depends on the number of boxes filled in the first row. For the multiset partition $\{\{1\},\{1\},\{1\},\{2\},\{2\},\{2\}\}$, the shape would be $(5,2,1) /(2)$ because we need to fill six boxes, three of these in the first row; however we cannot have two equal odd sets in any row, which means that this partition does not contribute to the coefficient. Similarly, the partition $\{\{\{1,2\},\{1,2\},\{1,2\}\}$ would fill a shape $(2,2,1) /(2)$ (in this case there are no filled boxes in the first row), but since we cannot repeat sets of even size on any column this partition does not contribute to the coefficient.

The other two partitions contribute to the coefficient of $\tilde{s}_{(2,1)}$. Notice that we have ordered the sets $\{1\}<\{1,2\}<\{2\}$. The partition $\{\{\{1\},\{1\},\{1,2\},\{2\},\{2\}\}$ will fill tableaux of shape $(4,2,1) /(2)$ and the partition $\{\{\{1\},\{1,2\},\{1,2\},\{2\}\}$ will fill tableaux of shape $(3,2,1) /(2)$. Below we list the 8 tableaux that contribute to the coefficient.

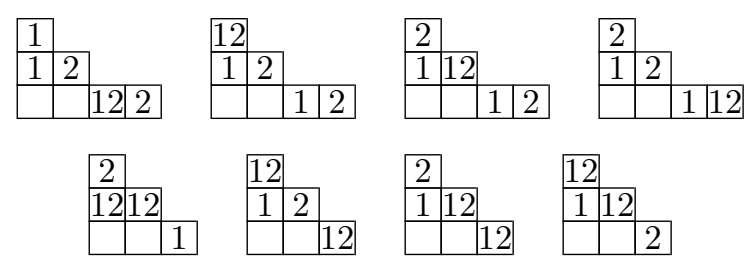

In [16, Lemma 5.10.1], Lascoux showed the following result. This result will serve as the starting point for our computations. In the following expressions, the notation $r \mid n$ indicates shorthand for " $r$ divides $n . "$

Proposition 5.4. For $r \geqslant 0, h_{0}\left[\Xi_{r}\right]=e_{0}\left[\Xi_{r}\right]=p_{0}\left[\Xi_{r}\right]=1$. In addition, for $n>0$,

$$
h_{n}\left[\Xi_{r}\right]=\delta_{r \mid n}, \quad p_{n}\left[\Xi_{r}\right]=r \delta_{r \mid n}, \quad e_{n}\left[\Xi_{r}\right]=(-1)^{r-1} \delta_{r=n} .
$$

We will need the evaluation and a combinatorial interpretation of $e_{\lambda}\left[\Xi_{\mu}\right]$ in order to make a connection with character symmetric functions. 
To extend this further, we determine the evaluation of an elementary symmetric function at $\Xi_{\mu}$. For a subset $S=\left\{i_{1}, i_{2}, \ldots, i_{|S|}\right\} \subseteq\{1,2, \ldots, \ell(\mu)\}$, let $\mu_{S}$ denote the sub-partition $\left(\mu_{i_{1}}, \mu_{i_{2}}, \ldots, \mu_{i_{|S|}}\right)$. This implies that

$$
e_{n}\left[\Xi_{\mu}\right]=\sum_{\substack{\alpha=w n \\ \ell(\alpha)=\ell(\mu)}} \prod_{i=1}^{\ell(\mu)} e_{\alpha_{i}}\left[\Xi_{\mu_{i}}\right]=\sum_{S:\left|\mu_{S}\right|=n} \prod_{i \in S} e_{\mu_{i}}\left[\Xi_{\mu_{i}}\right]=\sum_{S:\left|\mu_{S}\right|=n}(-1)^{n+|S|}
$$

where the sum is over all subsets $S \subseteq\{1,2, \ldots, \ell(\mu)\}$ such that $\left|\mu_{S}\right|=n$.

Definition 5.5. Define the set $\overline{\mathcal{C}}_{\lambda, \mu}$ to be the set of sequences $\left(S^{(1)}, S^{(2)}, \ldots, S^{(\ell(\lambda))}\right)$ where each $S^{(i)}$ is a subset such that $\left|\mu_{S^{(i)}}\right|=\lambda_{i}$.

Since $e_{\lambda}\left[\Xi_{\mu}\right]=e_{\lambda_{1}}\left[\Xi_{\mu}\right] e_{\lambda_{2}}\left[\Xi_{\mu}\right] \cdots e_{\lambda_{\ell(\lambda)}}\left[\Xi_{\mu}\right]$, it implies that we have the following Proposition for evaluating this expression.

Proposition 5.6. For partitions $\lambda$ and $\mu$,

$$
e_{\lambda}\left[\Xi_{\mu}\right]=\sum_{S^{(*)} \in \overline{\mathcal{C}}_{\lambda, \mu}}(-1)^{|\lambda|+\left|S^{(*)}\right|}
$$

where $\left|S^{(*)}\right|=\sum_{i=1}^{\ell(\lambda)}\left|S^{(i)}\right|$.

EXAMPLE 5.7 . Let $n=4$, then to evaluate $e_{4}\left[\Xi_{3211}\right]$ there are three subsets of parts of $(3,2,1,1)$ which sum to 4 , namely, $\{1,3\},\{1,4\}$ and $\{2,3,4\}$. The first two are counted with weight 1 and the third has weight -1 , hence $e_{4}\left[\Xi_{3211}\right]=1+1-1=1$.

To evaluate $e_{31}\left[\Xi_{3211}\right]$ we determine that $\overline{\mathcal{C}}_{31,3211}=\{(\{1\},\{3\}),(\{1\},\{4\})$, $(\{2,3\},\{3\}),(\{2,3\},\{4\}),(\{2,4\},\{3\}),(\{2,4\},\{4\})\}$. The first two of these have weight $(-1)^{|\lambda|+\left|S^{(*)}\right|}$ both equal to 1 and the last four have weight -1 hence $e_{31}\left[\Xi_{3211}\right]=-2$.

Let $\gamma^{(*)}=\left(\gamma^{(0)}, \gamma^{(1)}, \gamma^{(2)}, \ldots, \gamma^{(r)}\right)$ be sequences of partitions such that $\bigcup_{i=0}^{r} \gamma^{(i)}=$ $\mu$, then we may use Equation (1) to compute

$$
\frac{z_{\mu}}{z_{\gamma^{(0)}} z_{\gamma^{(1)}} z_{\gamma^{(2)}} \cdots z_{\gamma^{(r)}}}=\prod_{i \geqslant 1}\left(\begin{array}{c}
m_{i}(\mu) \\
m_{i}\left(\gamma^{(1)}\right), m_{i}\left(\gamma^{(2)}\right), \ldots, m_{i}\left(\gamma^{(r)}\right)
\end{array}\right)
$$

and the parts of $\gamma^{(0)}$ are determined from $\mu$ and all of the $\gamma^{(i)}$ for $1 \leqslant i \leqslant r$.

Now we will need to evaluate $E_{(\lambda \mid \tau), \mu}:=\left\langle p_{\mu}, h_{|\mu|-|\lambda|-|\tau|} h_{\lambda} e_{\tau}\right\rangle$ where $\lambda, \tau$ and $\mu$ are partitions. We do this by expanding the expression $h_{|\mu|-|\lambda|-|\tau|} h_{\lambda} e_{\tau}$. For each sequence of partitions $\gamma^{(*)}$ of length $\ell(\lambda)$ and each sequence of partitions $\nu^{(*)}$ of length $\ell(\tau)$, there will be one term in the sum coming from the expansion of the product of $h_{\lambda}$ and $e_{\tau}$. Expanding the expression for $E_{(\lambda \mid \tau), \mu}$, yields

$$
E_{(\lambda \mid \tau), \mu}=\sum_{\gamma^{(*)}, \nu^{(*)}} \operatorname{sgn}\left(\nu^{(*)}\right) \prod_{i=1}^{\mu_{1}}\left(\begin{array}{c}
m_{i}(\mu) \\
m_{i}\left(\gamma^{(1)}\right), \ldots, m_{i}\left(\gamma^{(\ell(\lambda))}\right), m_{i}\left(\nu^{(1)}\right), \ldots, m_{i}\left(\nu^{(\ell(\tau))}\right)
\end{array}\right)
$$

where the sum is over all sequences of partitions $\gamma^{(*)}=\left(\gamma^{(1)}, \gamma^{(2)}, \ldots, \gamma^{(\ell(\lambda))}\right)$ where $\gamma^{(j)} \vdash \lambda_{j}$ and $\nu^{(*)}=\left(\nu^{(1)}, \nu^{(2)}, \ldots, \nu^{(\ell(\tau))}\right)$ where $\nu^{(j)} \vdash \tau_{j}$ and

$$
\operatorname{sgn}\left(\nu^{(*)}\right)=(-1)^{\sum_{i}\left|\nu^{(i)}\right|+\ell\left(\nu^{(i)}\right)} .
$$


Note that we are using the convention that if $\bigcup_{i} \gamma^{(i)} \cup \bigcup_{i} \nu^{(i)}$ is not a subset of the parts of $\mu$ then the weight

$$
\prod_{i=1}^{\mu_{1}}\left(\begin{array}{c}
m_{i}(\mu) \\
m_{i}\left(\gamma^{(1)}\right), \ldots, m_{i}\left(\gamma^{(\ell(\lambda))}\right), m_{i}\left(\nu^{(1)}\right), \ldots, m_{i}\left(\nu^{(\ell(\tau))}\right)
\end{array}\right)
$$

is equal to 0 .

Proposition 5.8. For partitions $\lambda, \tau$ and $\mu$, let $\mathcal{F}_{\lambda, \tau}^{\mu}$ be the fillings of the diagram for the partition $\mu$ with $\lambda_{i}$ labels $i$ and $\tau_{j}$ labels $j^{\prime}$ such that all cells in a row are filled with the same label such that cells in any row are either all filled or all empty. For $F \in \mathcal{F}_{\lambda, \tau}^{\mu}$, the weight of the filling, wt $(F)$ is equal to -1 raised to the number of cells filled with primed labels plus the number of rows occupied by the primed labels. Then

$$
E_{(\lambda \mid \tau), \mu}=\sum_{F \in \mathcal{F}_{\lambda, \tau}^{\mu}} w t(F) .
$$

Proof. This is precisely the analogous statement to Proposition 28 of [23]. It follows because if we fix the sequences of partitions $\gamma^{(*)}$ and $\nu^{(*)}$ such that $\gamma^{(i)} \vdash \lambda_{i}$ and $\nu^{(j)} \vdash \tau_{j}$, then the quantity

$$
\prod_{i=1}^{\mu_{1}}\left(\begin{array}{c}
m_{i}(\mu) \\
m_{i}\left(\gamma^{(1)}\right), \ldots, m_{i}\left(\gamma^{(\ell(\lambda))}\right), m_{i}\left(\nu^{(1)}\right), \ldots, m_{i}\left(\nu^{(\ell(\tau))}\right)
\end{array}\right)
$$

is precisely the number of $F$ in $\mathcal{F}_{\lambda, \tau}^{\mu}$ with the rows filled according to the sequences of partitions $\gamma^{(*)}$ and $\nu^{(*)}$. The sign of a filling is constant on this set and is equal to $\operatorname{sgn}\left(\nu^{(*)}\right)$.

ExAMPLE 5.9. The following are all the possible fillings of the diagram $(3,3,2,2,1,1)$ with two 1's and two 1's such that the rows have the same labels.
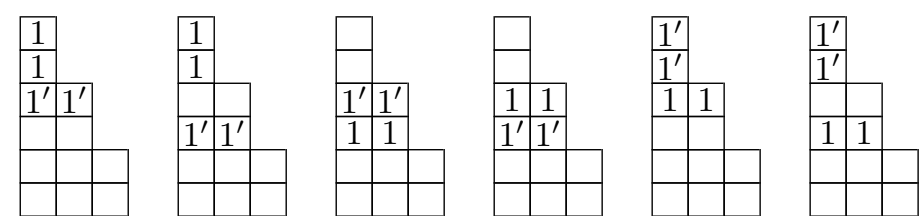

Since the weight of the filling is equal to the $(-1)$ raised to the number of cells plus the number of rows occupied by primed entries, the first four have weight -1 and the last two have weight 1 and hence

$$
E_{(2 \mid 2), 332211}=-2 .
$$

The rest of this section develops the combinatorial constructions required to show that the evaluations of $e_{\lambda}$ at roots of unity are correct.

We have previously used the notation $\pi H\left\{\left\{1^{\lambda_{1}}, 2^{\lambda_{2}} \ldots, \ell^{\lambda_{\ell}}\right\}\right.$ to indicate that $\pi$ is a multiset partition of a multiset. We will then use the notation $\pi \vdash\left\{\left\{1^{\lambda_{1}}, 2^{\lambda_{2}} \ldots, \ell^{\lambda_{\ell}}\right\}\right\}$ to indicate that $\pi$ is a set partition of a multiset, that is, $\pi=\left\{\left\{P^{(1)}, P^{(2)}, \ldots, P^{(\ell(\pi))}\right\}\right.$ where $P^{(1)} \uplus P^{(2)} \uplus \cdots \uplus P^{(\ell(\pi))}=\left\{\left\{1^{\lambda_{1}}, 2^{\lambda_{2}} \ldots, \ell^{\lambda_{\ell}}\right\}\right\}$ and each of the $P^{(i)}$ are sets (no repetitions allowed). It is possible that $\pi$ itself is a multiset since it is possible that $P^{(i)}=P^{(j)}$ when $i \neq j$. In this case we say that $\pi$ is a set partition of a multiset of content $\lambda$ (to differentiate from a multiset partition of a multiset).

Now we have previously defined $\tilde{m}(\pi)$ to be a partition representing the multiplicity of the sets that appear in $\pi$. Now define $\tilde{m}_{e}(\pi)$ be a partition representing the multiplicities of the sets with an even number of elements and $\tilde{m}_{o}(\pi)$ be a partition representing the multiplicities of the sets with an odd number of elements. 
EXAMPLE 5.10. Let $\lambda=(5,3,3,2,1)$ and then

$$
\pi=\{\{\{1,2,5\},\{1,2\},\{1,2\},\{1,3\},\{1,3\},\{3,4\},\{4\}\}\}
$$

is a set partition of the multiset $\left\{\left\{1^{5}, 2^{3}, 3^{3}, 4^{2}, 5\right\}\right.$. The corresponding partition $\tilde{m}(\pi)=(2,2,1,1,1)$ and $\tilde{m}_{e}(\pi)=(2,2,1)$ and $\tilde{m}_{o}(\pi)=(1,1)$. The sequence $\tilde{m}(\pi)$ is a partition of the length of $\pi$ and $\tilde{m}_{e}(\pi) \cup \tilde{m}_{o}(\pi)=\tilde{m}(\pi)$.

DEFINITION 5.11. For partitions $\lambda$ and $\mu$, let $\overline{\mathcal{P}}_{\lambda \mu}$ be the set of pairs $(\pi, T)$ where $\pi$ is a set partition of the multiset $\left\{1^{\lambda_{1}}, 2^{\lambda_{2}}, \ldots, \ell(\lambda)^{\lambda_{\ell(\lambda)}}\right\}$ and $T$ is a filling of some of the rows of the diagram for $\mu$ with content $\gamma=\tilde{m}_{e}(\pi)$ consisting of labels $\left\{\left\{1^{\gamma_{1}}, 2^{\gamma_{2}}, \cdots, \ell(\gamma)^{\gamma_{\ell(\gamma)}}\right\}\right.$ and some rows filled with content $\tau=\tilde{m}_{o}(\pi)$ consisting of primed labels $\left\{\left\{1^{\prime \tau_{1}}, 2^{\prime \tau_{2}}, \ldots, \ell(\tau)^{\prime \ell(\tau)}\right\}\right.$. The weight, wt, of a pair $(\pi, T)$ will be either \pm 1 and is equal to -1 raised to the number of primed labels plus the number of rows those labels occupy.

ExAmple 5.12. Consider the set $\overline{\mathcal{P}}_{(3,1),(3,3,2,2,1,1)}$ that consists of the following 12 pairs of set partitions and fillings

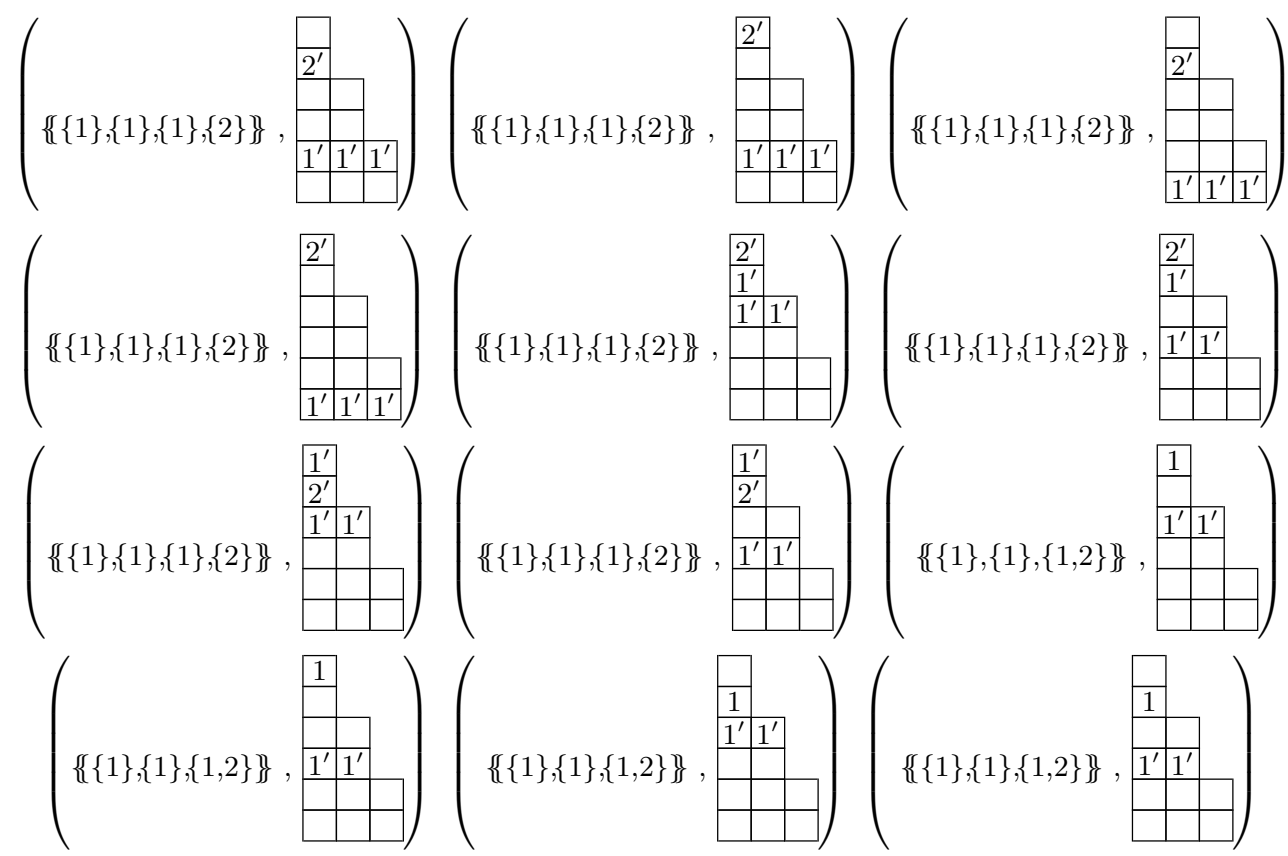

The first four of these pairs have weight +1 and the remaining eight have weight -1 .

With these definitions, we can use Proposition 5.8 to state that

$$
\sum_{\pi \vdash\left\{11^{\lambda_{1}}, 2^{\lambda_{2}}, \ldots, \ell^{\left(\lambda_{\ell}\right)}\right\}} E_{\left(\tilde{m}_{e}(\pi) \mid \tilde{m}_{o}(\pi)\right), \mu}=\sum_{F \in \overline{\mathcal{P}}_{\lambda, \mu}} w t(F) .
$$

Next we define a set $\overline{\mathcal{T}}_{\lambda, \mu}$, this set is defined in a similar way as $\overline{\mathcal{P}}_{\lambda, \mu}$, with the main difference that now the tableaux will contain the sets that make up the parts of $\pi$.

Definition 5.13. For partitions $\lambda$ and $\mu$ let $\overline{\mathcal{T}}_{\lambda, \mu}$ be the fillings of some of the cells of the diagram of the partition $\mu$ with subsets of $\{1,2, \ldots, \ell(\lambda)\}$ such that the total content of the filling is $\left\{\left\{1^{\lambda_{1}}, 2^{\lambda_{2}}, \ldots, \ell(\lambda)^{\left.\lambda_{\ell(\lambda)}\right\}}\right.\right.$ and such that all cells in the same row have the same subset of entries. We will define the weight of one of these fillings to be -1 to the power of the size of $\lambda$ plus the number of rows whose cells are occupied by a set of odd size (this is also equal to the number of cells plus the number of rows occupied by the sets of odd size). 
EXAMPLE 5.14. The following 12 tableaux are the elements of $\overline{\mathcal{T}}_{(3,1),(3,3,2,2,1,1)}$.
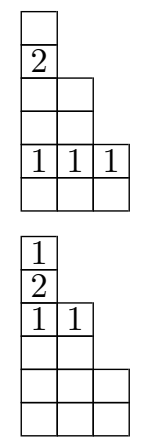
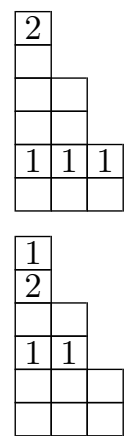
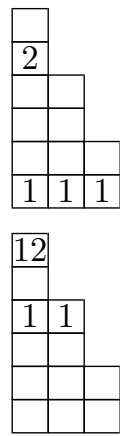
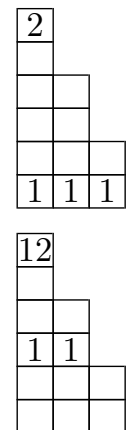
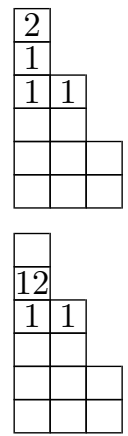
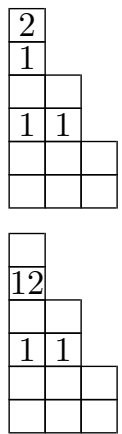

The fillings listed above are in the same order as their isomorphism with the set of pairs $\overline{\mathcal{P}}_{(3,1),(3,3,2,2,1,1)}$ from Example 5.12. As in that case we have that, the first four of these pairs have weight +1 and the remaining eight have weight -1 .

The following result should be clear from the definitions listed above and the examples we have presented.

LEMMA 5.15. There is a bijection between the sets $\overline{\mathcal{P}}_{\lambda, \mu}$ and $\overline{\mathcal{T}}_{\lambda, \mu}$ and $\overline{\mathcal{C}}_{\lambda, \mu}$ that preserves the weight.

Corollary 5.16. For partitions $\lambda$ and $\mu$,

$$
e_{\lambda}\left[\Xi_{\mu}\right]=\sum_{\pi \vdash\left\{\left\{1^{\lambda_{1}}, 2^{\lambda_{2}}, \ldots, \ell^{\lambda_{\ell}}\right\}\right.} H_{\left(\tilde{m}_{e}(\pi) \mid \tilde{m}_{o}(\pi)\right), \mu} .
$$

EXAMPLE 5.17. There are only three set partitions of $\left\{\left\{1^{3}, 2^{2}\right\}\right.$. These are

$$
\{\{\{1\},\{1\},\{1\},\{2\},\{2\}\},\{\{\{1\},\{1\},\{1,2\},\{2\}\},\{\{\{1\},\{1,2\},\{1,2\}\}\} .
$$

Corollary 5.16 states that

$$
e_{32}\left[\Xi_{\mu}\right]=E_{(\cdot \mid 32), \mu}+E_{(1 \mid 21), \mu}+E_{(2 \mid 1), \mu} .
$$

REMARK 5.18. The expressions $H_{(\lambda, \tau), \mu}$ implies that we could define symmetric functions $\tilde{h}_{(\lambda \mid \tau)}$ with the property $\tilde{h}_{(\lambda \mid \tau)}\left[\Xi_{\mu}\right]=E_{(\lambda \mid \tau), \mu}=\left\langle h_{|\mu|-|\lambda|-|\tau|} h_{\lambda} e_{\tau}, p_{\mu}\right\rangle$. Some of the results we present in this paper can be generalized to elements $\tilde{h}_{(\lambda \mid \tau)}$ which form a spanning set, but not a basis. This set of elements featured heavily in the thesis of Arash Islami in a project to develop formulae for a character basis for the hyperoctahedral group [14].

\section{REFERENCES}

[1] Sami H. Assaf and David E. Speyer, Specht modules decompose as alternating sums of restrictions of Schur modules, Proc. Amer. Math. Soc. 148 (2020), no. 3, 1015-1029.

[2] Cristina M. Ballantine and Rosa C. Orellana, A combinatorial interpretation for the coefficients in the Kronecker product $s_{(n-p, p)} * s_{\lambda}$, Sém. Lothar. Combin. 54A $(2005 / 07)$, Paper no. Art. B54Af (29 pages).

[3] Chris Bowman, Maud De Visscher, and John Enyang, The lattice permutation condition for Kronecker tableaux, arXiv preprint, https://arxiv.org/abs/1812.09175, 2018.

[4] Christopher Bowman, Maud De Visscher, and Rosa Orellana, The partition algebra and the Kronecker coefficients, Trans. Amer. Math. Soc. 367 (2015), no. 5, 3647-3667.

[5] Emmanuel Briand, Rosa Orellana, and Mercedes Rosas, Reduced Kronecker coefficients and counter-examples to Mulmuley's strong saturation conjecture SH, Comput. Complexity 18 (2009), no. 4, 577-600, With an appendix by Ketan Mulmuley.

[6] - The stability of the Kronecker product of Schur functions, J. Algebra 331 (2011), 11-27.

[7] Philip H. Butler and Roger C. King, The symmetric group: characters, products and plethysms, J. Mathematical Phys. 14 (1973), 1176-1183. 
[8] Thomas Church, Jordan S. Ellenberg, and Benson Farb, FI-modules and stability for representations of symmetric groups, Duke Math. J. 164 (2015), no. 9, 1833-1910.

[9] Thomas Church and Benson Farb, Representation theory and homological stability, Adv. Math. 245 (2013), 250-314.

[10] Inna Entova Aizenbud, Deligne categories and reduced Kronecker coefficients, J. Algebraic Combin. 44 (2016), no. 2, 345-362.

[11] Georg F. Frobenius, Über die Charaktere der symmetrischen Gruppe, Königliche Akademie der Wissenschaften, 1900.

[12] Adriano M. Garsia and Jeffrey Remmel, Shuffles of permutations and the Kronecker product, Graphs Combin. 1 (1985), no. 3, 217-263.

[13] Darij Grinberg and Victor Reiner, Hopf algebras in combinatorics, arXiv preprint, https:// arxiv.org/abs/1409.8356, 2014.

[14] Arash Islami, Symmetric functions as Characters of Hyperoctahedral Group, Ph.D. thesis, York University, 2020.

[15] Roger C. King, Branching rules for $G L(N) \supset \Sigma_{m}$ and the evaluation of inner plethysms, J. Mathematical Phys. 15 (1974), 258-267.

[16] Alain Lascoux, Symmetric functions, http://www.emis.de/journals/SLC/wpapers/ s68vortrag/ALCoursSf2.pdf, 2001.

[17] Dudley E. Littlewood, Products and plethysms of characters with orthogonal, symplectic and symmetric groups, Canadian J. Math. 10 (1958), 17-32.

[18] Ian G. Macdonald, Symmetric functions and Hall polynomials, second ed., Oxford Classic Texts in the Physical Sciences, The Clarendon Press, Oxford University Press, New York, 2015, With contribution by A. V. Zelevinsky and a foreword by Richard Stanley, Reprint of the 2008 paperback edition [ MR1354144].

[19] Francis D. Murnaghan, The characters of the symmetric group, Amer. J. Math. 59 (1937), no. 4 $739-753$.

[20] _ The analysis of the Kronecker product of irreducible representations of the symmetric group, Amer. J. Math. 60 (1938), no. 3, 761-784.

[21] _ On the analysis of the Kronecker product of irreducible representations of $S_{n}$, Proc. Nat. Acad. Sci. U.S.A. 41 (1955), 515-518.

[22] Kyo Nishiyama, Restriction of the irreducible representations of $G L_{n}$ to the symmetric group $S_{n}$, homepage preprint 2021-02-01, http://rtweb.math.kyoto-u.ac.jp/home_kyo/preprint/ glntosn.pdf, 2000.

[23] Rosa Orellana and Mike Zabrocki, Characters of the symmetric group as symmetric functions, arXiv preprint, https://arxiv.org/abs/1605.06672, 2016.

[24] _ , Products of symmetric group characters, J. Combin. Theory Ser. A 165 (2019), 299324.

[25] Bruce E. Sagan, The symmetric group: representations, combinatorial algorithms, and symmetric functions, vol. 203, Springer Science \& Business Media, 2013.

[26] Sage-Combinat community, Sage-Combinat: enhancing Sage as a toolbox for computer exploration in algebraic combinatorics, 2008, http://combinat.sagemath.org.

[27] Thomas Scharf and Jean-Yves Thibon, A Hopf-algebra approach to inner plethysm, Advances in Mathematics 104 (1994), no. 1, 30-58.

[28] Thomas Scharf, Jean-Yves Thibon, and Brian G Wybourne, Generating functions for stable branching coefficients $U(n) \downarrow S_{n}, O(n) \downarrow S_{n}$ and $O(n-1) \downarrow S_{n}$, J. Phys. A 30 (1997), no. 19, 6963-6975.

[29] Wilhelm Specht, Die charaktere der symmetrischen gruppe, Math. Z. 73 (1960), 312-329.

[30] Richard P. Stanley, Enumerative combinatorics. Vol. 2, Cambridge Studies in Advanced Mathematics, vol. 62, Cambridge University Press, Cambridge, 1999.

[31] William Stein and David Joyner, Sage: System for algebra and geometry experimentation, Acm Sigsam Bulletin 39 (2005), no. 2, 61-64.

[32] Moss E. Sweedler, Hopf algebras, Mathematics Lecture Note Series, W. A. Benjamin, Inc., New York, 1969.

[33] Alfred Young, The collected papers of Alfred Young (1873-1940), Mathematical Expositions, no. 21, University of Toronto Press, Toronto, Ont., Buffalo, N. Y., 1977. 
Rosa Orellana \& Mike Zabrocki

Rosa Orellana, Dartmouth College, Mathematics Department, Hanover, NH 03755, USA E-mail : rosa.c.orellana@dartmouth.edu

Mike Zabrocki, Department of Mathematics and Statistics, York University, Toronto, Ontario M3J 1P3, Canada

E-mail : zabrocki@mathstat.yorku.ca 